\title{
THE GENERALIZED DOWLING LATTICES
}

\author{
PHIL HANLON
}

\begin{abstract}
In this paper we study a new class of lattices called the generalized Dowling lattices. These lattices are parametrized by a positive integer $n, \mathrm{a}$ finite group $G$, and a meet sublattice $K$ of the lattice of subgroups of $G$. For an appropriate choice of $K$ the generalized Dowling lattice $D_{n}(G, K)$ agrees with the ordinary Dowling lattice $D_{n}(G)$. For a different choice of $K$, the generalized Dowling lattices are the lattice of intersections of a set of subspaces in complex space. The set of subspaces, defined in terms of a representation of $G$, generalizes the thick diagonal in $\mathbb{C}^{n}$.

We compute the Möbius function and characteristic polynomial of the lattice $D_{n}(G, K)$ along with the homology of $D_{n}(G, K)$ in terms of the homology of $K$. We go on to compute the character of $G$ wr $S_{n}$ acting on the homology of $D_{n}(G, K)$. This computation provides a nontrivial generalization of a result due to Stanley concerning the character of $S_{n}$ acting on the top homology of the partition lattice.
\end{abstract}

\section{INTRODUCTION}

In this paper we study a family of lattices which we call generalized Dowling lattices. These lattices, denoted $D_{n}(G, K)$, are indexed by a positive integer $n$, a finite group $G$, and a meet sublattice ${ }^{1} K$ of the lattice of subgroups of $G$. For an appropriate choice of $K, D_{n}(G, K)$ is the ordinary Dowling lattice of rank $n$ based on the finite group $G$. In this paper we study combinatorial properties of the generalized Dowling lattices and their order complexes. In particular, we compute the homology of $D_{n}(G, K)$ in terms of the homology off $K$.

The generalized Dowling lattices have a very rich combinatorial structure. Unless $K$ is trivial, $D_{n}(G, K)$ is not ranked hence is not supersolvable, geometric, or even Cohen-Macaulay. Nonetheless, all of the well-known combinatorial and homological properties of the Dowling lattices have elegant analogues in the generalized Dowling lattice case. Because the generalized Dowling lattices are not Cohen-Macaulay, these properties cannot be proved using classical combinatorial methods. In this paper we use a mixture of combinatorial and

Received by the editors May 1, 1989.

1980 Mathematics Subject Classification (1985 Revision). Primary 05B35.

The author is grateful to the Sloan Foundation, the IHES, the University of Michigan, and the National Science Foundation for partial support of this work.

"A "meet sublattice" $M$ of a lattice $L$ refers to a lattice $M$ contained in $L$ with the property that the meet in $M$ agrees with the meet in $L$. 
homological methods to compute $H_{*}\left(D_{n}(G, K)\right)$. In particular, we devise a spectral sequence method which effectively separates $H_{*}\left(D_{n}(G, K)\right)$ into two parts-a part coming from $H_{*}(K)$ and a part coming from the homology of the ordinary Dowling lattices.

The wreath product group $G \mathrm{wr} S_{n}$ acts as a group of automorphisms of $D_{n}(G, K)$. The spectral sequence used to compute $H_{*}\left(D_{n}(G, K)\right)$ is $\left(G \mathrm{wr} S_{n}\right)$ -equivariant which allows us to compute the character of $G \mathrm{wr} S_{n}$ on $H_{*}\left(D_{n}(G, K)\right)$. We prove a generalization of Stanley's result which expresses the action of $S_{n}$ on the top homology of the partition lattice in terms of induced characters.

There is a second choice of $K$ for which the $D_{n}(G, K)$ specialize to an interesting class of lattices. These specializations have a geometric significancetheir homology groups determine the homology of a natural affine variety in complex space. This variety is a generalization of the variety $\mathbb{C}^{n} \backslash \Delta$, where $\Delta$ is the thick diagonal, $\Delta=\left\{\left(v_{1}, \ldots, v_{n}\right): v_{i}=v_{j}\right.$ some $\left.i<j\right\}$. This generalized variety begins with a faithful representation $\varphi: G \rightarrow \mathrm{GL}(V)$ of a finite group $G$. For $i \leq j$ and $g \in G$ define $\mathscr{H}_{i, j, g}$ to be the subspace of $V^{n}$ given by

$$
\mathscr{H}_{i, j, g}=\left\{\left(v_{1}, \ldots, v_{n}\right) \in V^{n}: v_{j}=\varphi(g) v_{i}\right\} .
$$

For $i \neq j, \mathscr{H}_{i, j, g}$ has codimension equal to $\operatorname{dim}(V)$. For $i=j$ however, $\mathscr{H}_{i, j, g}$ can have smaller codimension. Let $\mathscr{S}_{n}(\varphi)$ denote the set of subspaces

$$
\mathscr{S}_{n}(\varphi)=\left\{\mathscr{H}_{i, j, g}: i \leq j, g \in G\right\} \backslash\left\{\mathscr{H}_{i, i, e}: 1 \leq i \leq n\right\} .
$$

Note that $\mathscr{H}_{i, j, g}=V^{n}$ iff $i=j$ and $g=e$. Thus the exclusion of the set $\left\{\mathscr{H}_{i, i, e}: 1 \leq i \leq n\right\}$ insures that $V^{n}$ is not in $\mathscr{S}_{n}(\varphi)$. Let $\widehat{\Delta}$ be the union of the subspaces in $\mathscr{S}_{n}(\varphi)$ and let $\Lambda_{n}(\varphi)$ be $V^{n} \backslash \widehat{\Delta}$. It is easy to see that $\Lambda_{n}(\varphi)=\mathbf{C}^{n} \backslash \Delta$ when the group $G$ is trivial, so one can think of $\Lambda_{n}(\varphi)$ as an affine variety which generalizes $\mathbf{C}^{n} \backslash \Delta$.

Using results of Goresky and Macpherson (see [8]) one can compute the homology of $\Lambda_{n}(\varphi)$ in terms of the homology of the lattice of intersections of the subspaces in $\mathscr{S}_{n}(\varphi)$. We will show that this lattice of intersections is $D_{n}(G, K)$ for an appropriate $K$. So the computation of $H_{*}\left(D_{n}(G, K)\right)$ determines the homology of the affine variety $\Lambda_{n}(\varphi)$.

The paper is organized in the following way. In $\S 2$ we define the generalized Dowling lattices and develop some of their more elementary combinatorial properties. In $\S 3$ we prove $D_{n}(G, K)$ is isomorphic to the lattice of intersections of the subspaces in $\mathscr{S}_{n}(\varphi)$. $\S 4$ contains the main results. We introduce our spectral sequence methods, use them to compute the homology of the generalized Dowling lattices, and apply that to the computation of $H_{*}\left(\Lambda_{n}(\varphi)\right)$. In $\S 5$ we conclude by computing the character values for the action of the wreath product group $G$ wr $S_{n}$ on the homology of $D_{n}(G, K)$. 
The author would like to thank Mark Goresky for many helpful conversations during the preparation of this manuscript.

\section{THE COMBINATORIAL THEORY}

2.1. $K$-digraphs. Let $L$ be a finite lattice. A closure operator on $L$ is a map $x \rightarrow \bar{x}$ from $L$ to $L$ which satisfies:

(a) $\bar{x} \geq x$ for all $x \in L$.

(b) $\overline{\bar{x}}=\bar{x}$ for all $x \in L$.

(c) If $x \leq y$ then $\bar{x} \leq \bar{y}$ for all $x, y \in L$.

We will also assume the condition $\overline{0}=0$ (where 0 denotes the minimal element of $L$ ). If $x \rightarrow \bar{x}$ is a closure operator on $L$ then we let $\bar{L}$ denote the set of closed elements of $L$, i.e.,

$$
\bar{L}=\{x \in L: \bar{x}=x\} .
$$

Proposition 2.1.1. Let $x \rightarrow \bar{x}$ be a closure on $L$ and let $x, y \in \bar{L}$. Then $x \wedge y$ is in $\bar{L}$.

Proof. For $x, y \in \bar{L}$ we have

$$
\overline{x \wedge y} \leq \bar{x}=x \text { and } \overline{x \wedge y} \leq \bar{y}=y .
$$

So,

$$
\overline{x \wedge y} \leq x \wedge y \leq \overline{x \wedge y} .
$$

Proposition 2.1.1 shows that $\bar{L}$ has a meet operation. Also it has a maximal element (the maximal element of $L$ ) and so it is a lattice. In general it is not a sublattice of $L$ as only the meet operations in $L$ and $\bar{L}$ agree.

Let $G$ be a finite group with identity $e$ and let $L(G)$ denote the lattice of subgroups of $G$. We let $E$ denote the trivial subgroup $E=\{e\}$. Assume that $H \rightarrow \bar{H}$ is a conjugation-invariant closure operator on $L(G)$. In other words, the map $H \rightarrow \bar{H}$ satisfies

$$
\overline{g^{-1} H g}=g^{-1} \bar{H} g
$$

for $g \in G$ and $H \in L(G)$. We let $K$ denote the lattice $\overline{L(G)}$ of closed subgroups in $L(G)$. By the conjugation-invariance of the closure operator we have that $G$ acts as a group of automorphisms of $K$.

Definition 2.1.2. A complete $K$-digraph with vertex set $S$ is an edge-labelled digraph with an edge directed from $s$ to $t$ for every pair $(s, t) \in S \times S$. The edge from $s$ to $t$ is labelled with a right coset $H_{s, t}$ of some subgroup in $K$. Moreover the following consistency condition $(*)$ must be satisfied:

For every $(r, s, t) \in S^{3}$ we have

$$
H_{s, t} H_{r, s}=H_{r, t},
$$

where $A B$ denotes $\{\alpha \beta: \alpha \in A, \beta \in B\}$.

Condition $(*)$ is a strong constraint as the next proposition shows. 
Proposition 2.1.3. Let $\delta$ be a complete $K$-digraph with vertex set $S$ and with edge labels $H_{u, v}$.

(a) For each $u \in S, H_{u, u}$ is a subgroup in $K$.

(b) Let $u, v \in S$. Then $H_{u, v}$ is a right coset of the subgroup $H_{u, u}$.

(c) Let $u, v \in S$. Then $H_{v, u}=\left\{\alpha^{-1}: \alpha \in H_{u, v}\right\}$.

(d) Let $u, v \in S$ and let $\gamma$ be any element of $H_{u, v}$. Then

$$
H_{v, v}=\gamma H_{u, u} \gamma^{-1} \text {. }
$$

Proof. We first prove (a). Choosing $r=s=t=u$ in $(*)$ gives that $H_{u, u}$ is closed under multiplication, hence is a subgroup.

To prove (b) first choose $r=s=u$ and $t=v$ in (*). We obtain

$$
H_{u, v} H_{u, u}=H_{u, v} .
$$

It follows that $H_{u, v}$ is a union of right cosets of $H_{u, u}$. Next choose $r=t=u$ and $s=v$ in $(*)$. This gives

$$
H_{v, u} H_{u, v}=H_{u, u}
$$

so $\left|H_{u, v}\right| \leq\left|H_{u, u}\right|$. Thus $H_{u, v}$ is a single right coset of $H_{u, u}$.

We next prove (c). As above we have

$$
H_{v, u} H_{u, v}=H_{u, u}
$$

so (1) $\left|H_{v, u}\right| \leq\left|H_{u, u}\right|$ and (2) for some $\gamma \in H_{u, v}$ we have $\gamma^{-1} \in H_{v, u}$. Choosing $r=u$ and $s=t=v$ in (*) we obtain

$$
H_{u, u} H_{v, u}=H_{v, u} \text {. }
$$

So $H_{v, u}$ is a left coset of $H_{u, u}$ (here using (1)). By fact (2) we have $H_{v, u}=$ $H_{u, u} \gamma^{-1}$ from which (c) follows.

Lastly we prove (d). By (c) we know that $\gamma^{-1}$ is in $H_{v, u}$. Hence by $(*)$ we have that

$$
H_{u, u} \gamma^{-1} \subseteq H_{v, u} .
$$

Applying $(*)$ again we have that

$$
\gamma H_{u, u} \gamma^{-1} \subseteq H_{v, v} .
$$

Reversing the roles of $u$ and $v$ in the above argument shows that

$$
\gamma^{-1} H_{v, v} \gamma \subseteq H_{u, u}
$$

which completes the proof of $(\mathrm{d})$.

Proposition 2.1.3 gives a simple method for constructing all the complete $K$-digraphs with vertex set $S$.

Step 1. Fix a point $u \in S$ and choose a subgroup $H_{u, u} \in K$. Let $R$ be a set of coset representatives for $H_{u, u}$ in $G$. 
Step 2. For each $v \neq u$ in $S$ choose a representative $r(u, v) \in R$. Once these (independent) choices have been made we are constrained, by Proposition 2.1.3, to define $H_{v, w}$ to be

$$
H_{v, w}=r(u, w) H_{u, u} r(u, v)^{-1} .
$$

In particular, the number of complete $K$-digraphs which have subgroups conjugate to $H$ labelling its loops is $(|G| /|H|)^{|S|-1}$.

Definition 2.1.4. A $K$-digraph is a digraph each of whose weakly connected components is a complete $K$-digraph.

Let $\Omega_{n}(G, K)$ denote the set of $K$-digraphs with vertex set $\{1,2, \ldots, n\}$. Given a $K$-digraph $\delta \in \Omega_{n}(G, K)$ we define its edge labels by: $H_{u, v}$ is the actual label on the edge from $u$ to $v$ if this edge exists in $\delta$ and is the empty set if there is no edge from $u$ to $v$ in $\delta$.

Define a partial ordering $\leq$ on $\Omega_{n}(G, K)$ in the following way. Let $\delta_{1}$ and $\delta_{2}$ be in $\Omega_{n}(G, K)$ and let $H_{u, v}^{(i)}$ be the edge labels on $\delta_{i}$. Then we say $\delta_{1} \leq \delta_{2}$ iff $H_{u, v}^{(1)} \subseteq H_{u, v}^{(2)}$ for all $u$ and $v$.

It is obvious that $\leq$ is a partial ordering on $\Omega_{n}(G, K)$. The poset $\Omega_{n}(G, K)$ has a unique minimal element 0 which is the digraph whose only edges are a loop at each point labelled $E$. Also $\Omega_{n}(G, K)$ has a unique maximal element 1 which is the complete digraph with every edge labelled $G$.

We will show that $\Omega_{n}(G, K)$ is a lattice. Since $\Omega_{n}(G, K)$ has a maximal element, it is enough to show that every pair of elements has a greatest lower bound. We do this by explicitly describing the greatest lower bound. This description makes use of the following fact which is easy to prove.

Proposition 2.1.5. Let $A$ and $B$ be subgroups of a finite group $G$ and let $\alpha A$ and $\beta B$ be right cosets of these subgroups. Then $(\alpha A) \cap(\beta B)$ is either empty or it is a right coset of $(A \cap B)$.

Definition 2.1.6. Let $\delta_{1}$ and $\delta_{2}$ be in $\Omega_{n}(G, K)$, and let $H_{u, v}^{(i)}$ be the edge labels in $\delta_{i}$. Recall that $H_{u, v}^{(i)}=\varnothing$ if there is no edge from $u$ to $v$ in $\delta_{i}$. Define $\delta_{1} \wedge \delta_{2}$ to be the digraph with edge labels $H_{u, v}$ defined by

$$
H_{u, v}=H_{u, v}^{(1)} \cap H_{u, v}^{(2)} .
$$

If $H_{u, v}=\varnothing$ then we interpret that to mean that there is no edge from $u$ to $v$ in $\delta_{1} \wedge \delta_{2}$.

Lemma 2.1.7. The digraph $\delta_{1} \wedge \delta_{2}$ defined above is in $\Omega_{n}(G, K)$.

Proof. By Propositions 2.1.1 and 2.1.5, the label on every edge of $\delta_{1} \wedge \delta_{2}$ is a right coset of some subgroup in $K$. Also by Proposition 2.1.1 there is a loop at every point of $\delta_{1} \wedge \delta_{2}$ labelled with some subgroup in $K$. So it suffices to verify condition $(*)$. 
Let $(r, s, t) \in[n]^{3}$ and suppose there are edges from $r$ to $s$ and from $s$ to $t$ in $\delta_{1} \wedge \delta_{2}$. Let $\gamma(r, s)$ be an element of

$$
H_{r, s}=H_{r, s}^{(1)} \cap H_{r, s}^{(2)}
$$

and let $\gamma(s, t)$ be an element of $H_{s, t}=H_{s, t}^{(1)} \cap H_{s, t}^{(2)}$. The following equations follow immediately from Proposition 2.1.3:

$$
\begin{gathered}
H_{r, s}^{(i)}=\gamma(r, s) H_{r, r}^{(i)}, \quad i=1,2, \\
H_{s, t}^{(i)}=\gamma(s, t) H_{s, s}^{(i)}, \quad i=1,2, \\
H_{s, s}^{(i)}=\gamma(r, s) H_{r, r}^{(i)} \gamma(r, s)^{-1}, \quad i=1,2 .
\end{gathered}
$$

So

$$
\begin{aligned}
H_{r, t}^{(i)} & =H_{s, t}^{(i)} H_{r, s}^{(i)} \\
& =\gamma(s, t) \gamma(r, s) H_{r, r}^{(i)} \gamma(r, s)^{-1} \gamma(r, s) H_{r, r}^{(i)} \\
& =\gamma(s, t) \gamma(r, s) H_{r, r}^{(i)}, \quad i=1,2 .
\end{aligned}
$$

Thus,

$$
H_{r, t}=H_{r, t}^{(1)} \cap H_{r, t}^{(2)}=\gamma(s, t) \gamma(r, s)\left(H_{r, r}^{(1)} \cap H_{r, r}^{(2)}\right) .
$$

Also from equations (E1)-(E3) we have

$$
H_{r, s}^{(1)} \cap H_{r, s}^{(2)}=\gamma(r, s)\left(H_{r, r}^{(1)} \cap H_{r, r}^{(2)}\right)
$$

and

So

$$
H_{s, t}^{(1)} \cap H_{s, t}^{(2)}=\gamma(s, t) \gamma(r, s)\left(H_{r, r}^{(1)} \cap H_{r, r}^{(2)}\right) \gamma(r, s)^{-1} .
$$

$$
\begin{aligned}
H_{s, t} H_{r, s} & =\left(H_{s, t}^{(1)} \cap H_{s, t}^{(2)}\right)\left(H_{r, s}^{(1)} \cap H_{r, s}^{(2)}\right) \\
& =\gamma(s, t) \gamma(r, s)\left(H_{r, r}^{(1)} \cap H_{r, r}^{(2)}\right)=H_{r, t}^{(1)} \cap H_{r, t}^{(2)}
\end{aligned}
$$

and this verifies condition $(*)$.

Given that $\delta_{1} \wedge \delta_{2}$ is in $\Omega_{n}(G, K)$ it is immediate that $\delta_{1} \wedge \delta_{2}$ is a greatest lower bound for $\delta_{1}$ and $\delta_{2}$. We have proved the following theorem.

Theorem 2.1.8. The poset $\Omega_{n}(G, K)$ is a lattice. For $\delta_{1}, \delta_{2} \in \Omega_{n}(G, K)$ the meet of $\delta_{1}$ and $\delta_{2}$ is the digraph $\delta_{1} \wedge \delta_{2}$ defined above.

It should be noted that the join operation in $\Omega_{n}(G, K)$ is much more difficult to understand. We know of no simple description of this operation.

Definition 2.1.9. Let $L$ be a lattice with minimal element 0 . A rank function $r$ on $L$ is a strictly increasing function, $r: L \rightarrow \mathbb{N}$, which satisfies:

(1) $r(0)=0$.

(2) For all $x, y \in L$,

$$
r(x \vee y)+r(x \wedge y) \leq r(x)+r(y) .
$$


For example, if $L$ is geometric then we can take $r(x)$ to be the length of a maximal chain from 0 to $x$. The lattices we deal with will not satisfy the chain condition so our rank functions will be defined in a different way.

Given a rank function $r$ on $L$ we define the characteristic polynomial of $L$ with respect to $r$ by

$$
\chi_{r}(L ; \lambda)=\sum_{x \in L} \mu_{L}(0, x) \lambda^{r(1)-r(x)} .
$$

In this definition, $\mu_{L}$ denotes the Möbius function of $L$ (see Rota [15]) and 1 denotes the maximal element of $L$. Usually the rank function $r$ will be understood and we will write $\chi(L ; \lambda)$ in place of $\chi_{r}(L ; \lambda)$.

Henceforth assume that $r$ is a conjugation-invariant rank function on $K$, i.e., a rank function satisfying $r\left(g^{-1} H g\right)=r(H)$ for $g \in G$ and $H \in K$.

Definition 2.1.10. Define a rank function $\rho=\rho_{r}$ on $\Omega_{n}(G, K)$ in the following way. Let $\delta \in \Omega_{n}(G, K)$ and let $\delta_{1}, \ldots, \delta_{l}$ be the connected components of $\delta$. Let $a_{i}$ denote the size of $\delta_{i}$ and let $H_{i}$ be the subgroup labelling an arbitrary chosen point of $\delta_{i}$. Then

$$
\rho(\delta)=(n-l)+\sum_{i=1}^{l}\left\{r\left(H_{i}\right)+\left(a_{i}-1\right) r(G)\right\} .
$$

Although it is not obvious, $\rho$ is a rank function on $\Omega_{n}(G, K)$. This fact is not important for what follows so we leave the proof to the reader. Our immediate goal is to compute the characteristic polynomial of $\Omega_{n}(G, K)$ (with respect to $\rho$ ) in terms of the characteristic polynomial of $K$ (with respect to $r$ ). For this computation we need the value of the Möbius function in $\Omega_{n}(G, K)$ which we will compute using the following result due to Crapo (see [4]).

Lemma 2.1.11 (Crapo's complementation theorem). Let $L$ be a finite lattice, let $x$ be an element of $L \backslash\{0,1\}$, and let $C_{x}$ be the set of complements of $x$ in $L$, i.e.,

$$
C_{x}=\{y \in L: x \vee y=1 \text { and } x \wedge y=0\} \text {. }
$$

Then,

$$
\mu_{L}(0,1)=\sum_{y_{1}, y_{2} \in C_{x}} \mu_{L}\left(0, y_{1}\right) \zeta\left(y_{1}, y_{2}\right) \mu_{L}\left(y_{2}, 1\right),
$$

where $\zeta\left(y_{1}, y_{2}\right)$ is 1 if $y_{1} \leq y_{2}$ and 0 otherwise.

We will apply Lemma 2.1.11 to prove the following result which gives the value of the Möbius function of $\Omega_{n}(G, K)$ in terms of the Möbius function of $K$.

Theorem 2.1.12. Let $G, K$, and $\Omega_{n}(G, K)$ be as above. Then

$$
\mu_{\Omega_{n}(G, K)}(0,1)=\mu_{K}(E, G)|G|^{n-1}(-1)^{n-1}(n-1) ! .
$$

Proof. We prove this by induction on $n$, the case $n=1$ being obvious. Assume $n$ is greater than 1 and that the theorem is known for values smaller than $n$. 
Let $x^{\prime}$ be the element of $\Omega_{n}(G, K)$ which has two connected components, $\delta_{0}$ consisting of the single point $\{n\}$ and $\delta_{1}$ consisting of $\{1,2, \ldots, n-1\}$. Let every edge of $x^{\prime}$ be labelled by $G$. We will apply Lemma 2.1 .11 to this choice of $x^{\prime}$.

Let $y$ be a complement to $x^{\prime}$.

Claim 1. Every loop of $y$ is labelled with $E$.

Proof. Let $u$ be a point of $y$ and suppose the loop at $u$ is labelled $H$. Let $z$ be the digraph whose only edges are the loops with every loop labelled $E$ except the loop at $u$ which is labelled $H$. Then

$$
z \leq x^{\prime} \wedge y=0
$$

so $H=E$. This proves Claim 1 .

By a similar argument it is possible to prove:

Claim 2. Suppose $1 \leq u<v \leq n-1$. Then there is no edge from $u$ to $v$ in $y$.

Since $x \vee y=1, y$ must have some edges other than loops. It follows from Claims 1 and 2 that $y$ must be of the form $y=A_{u, n, g}$, where $A_{u, n, g}$ has $n+2$ edges, $n$ loops labelled $E$, an edge from $u$ to $n$ labelled $E g$, and an edge from $n$ to $u$ labelled $E g^{-1}$. Thus

$$
\left|C_{x^{\prime}}\right|=(n-1)|G| .
$$

Observe that these complements $A_{u, n, g}$ are atoms in $\Omega_{n}(G, K)$ so they are incomparable and $\mu\left(0, A_{u, n, g}\right)=-1$. Consider the interval $\left[A_{u, n, g}, 1\right]$ in $\Omega_{n}(G, K)$. Let $\delta$ be a digraph in the interval with edge labels $H_{r, s}$. The following facts are immediate consequences of Proposition 2.1.3 and the definition of $A_{u, n, g}$ :

(1) $u$ and $n$ are in the same connected component of $\delta$.

(2) $H_{n, n}=g H_{u, u} g^{-1}$.

(3) For all $v \in[n], H_{v, n}=g H_{v, u}$.

It follows that the edges incident to $n$ and their labels are completely determined by the edges incident to $u$ and their labels. So the map $\delta \rightarrow \bar{\delta}$ from $\left[A_{u, n, g}, 1\right]$ to $\Omega_{n-1}(G, K)$ which removes the point $n$ is an isomorphism. Thus

$$
\mu\left(A_{u, n, g}, 1\right)=\mu_{\Omega_{n-1}(G, K)}(0,1) .
$$

Applying Lemma 2.1.11 and our induction hypothesis we have

$$
\begin{aligned}
\mu_{\Omega_{n}(G, K)}(0,1) & =(n-1)|G|\left\{(-1) \cdot\left(\mu_{K}(E, G)(-1)^{n-2}|G|^{n-2}(n-2) !\right)\right\} \\
& =\mu_{k}(E, G)(-1)^{n-1}|G|^{n-1}(n-1) !
\end{aligned}
$$

The next lemma will also be important in our computation of the characteristic polynomial of $\Omega_{n}(G, K)$. 
Lemma 2.1.13. Let $\delta$ be a complete $K$-digraph in $\Omega_{n}(G, K)$, let $u \in[n]$, and let $H$ be the subgroup labelling the loop at $u$ in $\delta$. Let $K^{\prime}$ be the interval $[E, H]$ in $K$. Then the interval $[0, \delta]$ in $\Omega_{n}(G, K)$ is isomorphic to the interval $[0,1]$ in $\Omega_{n}\left(H, K^{\prime}\right)$.

Proof. For each $s \in[n]$, let $\gamma_{u, s}$ be an element chosen from the coset which labels the edge from $u$ to $s$ in $\delta$. Define an isomorphism $\varphi$,

in terms of this choice of $\gamma_{r, s}$.

$$
\varphi:[0, \delta]_{\Omega_{n}(G, K)} \rightarrow[0,1]_{\Omega_{n}\left(H, K^{\prime}\right)},
$$

To define $\varphi$, let $\pi \in[0, \delta]$ and let $H_{r, s}$ be the edge labels of $\pi$. Define $\varphi(\pi)$ to have the same set of edges as $\pi$ but the label on the edge from $v$ to $w$ in $\varphi(\pi)$ is changed to $\gamma_{u, w}^{-1} H_{v, w} \gamma_{u, v}$. It is straightforward to check that

(1) $\varphi(\pi) \in \Omega_{n}\left(H, K^{\prime}\right)$, and

(2) $\varphi$ is a lattice isomorphism.

Theorem 2.1.14. Let $G, K$, and $\Omega_{n}(G, K)$ be as above. Then

$$
\lambda \chi_{\rho}\left(\Omega_{n}(G, K) ; \lambda\right)=\prod_{i=0}^{n-1}\left(\lambda \chi_{r}(K ; \lambda)-i|G|\right) .
$$

Proof. Let $j_{1}, \ldots, j_{n}$ be a sequence with $\sum i j_{i}=n$. We will first compute the contribution to $\chi_{\rho}\left(\Omega_{n}(G, K) ; \lambda\right)$ made by all terms $\mu_{\Omega_{n}(G, K)}(0, \delta) \lambda^{\rho(1)-\rho(\delta)}$, where $\delta$ has $j_{i}$ connected components of size $i$. We construct all such $\delta$ via the following procedure:

Step 1. Choose which points go in each component. This can be done in $n ! / \prod_{i}(i !)^{j_{i}} j_{i}$ ! ways.

Step 2. In each component $\delta_{s}$ choose a point $u_{s}$ and a subgroup $H_{s}$ to label the loop at $u_{s}$. For each $v \neq u_{s}$ in $\delta_{s}$ choose a coset $H_{u_{s}, v}$ of $H_{s}$ to label the edge $\varepsilon\left(u_{s}, v\right)$.

Once $H_{s}$ is chosen, this can be done in $\left(|G| /\left|H_{s}\right|\right)^{\left|\delta_{s}\right|-1}$ ways. For this $\delta$ we have that $1+\rho(1)-\rho(\delta)=\sum_{s}\left(r(g)-r\left(H_{s}\right)+1\right)$. The interval from 0 to $\delta$ is isomorphic to the direct product of the intervals from 0 to 1 in the lattices $\Omega_{\left|\delta_{s}\right|}\left(H_{s}, K_{s}\right)$, where $K_{s}$ is the interval $\left[E, H_{s}\right]$ in $K$ (by Lemma 2.1.13). So the contribution to the characteristic polynomial made by the digraphs constructed with these choices is

$$
\begin{gathered}
\frac{n !}{\lambda} \prod_{i} \frac{1}{(i !)^{j_{i}} j_{i} !} \prod_{s} \lambda^{r(G)-r\left(H_{s}\right)+1} \mu_{K}\left(0, H_{s}\right)(-1)^{\left|\delta_{s}\right|-1} \\
\cdot\left(\left|\delta_{s}\right|-1\right) !\left(\left|H_{s}\right|\left(\frac{|G|}{\left|H_{s}\right|}\right)\right)^{\left|\delta_{s}\right|-1} .
\end{gathered}
$$

Summing this over all possible $j_{1}, \ldots, j_{n}$ we obtain

$$
\lambda \chi_{\Omega_{n}(G, K)}(\lambda)=n !|G|^{n}(-1)^{n} \sum_{j_{1}, \ldots, j_{n}} \frac{1}{\prod_{i} i^{j_{i}} j_{i} !} \prod_{i}\left(\sum_{H \in K} \mu(0, H) \lambda^{r(G)-r(H)}\left(\frac{-\lambda}{|G|}\right)\right)^{j_{i}} .
$$


Since $n ! / \prod_{i} i^{j_{i}} j_{i}$ ! is the number of elements in the symmetric group $S_{n}$ with $j_{i}$ cycles of length $i$, we have

$$
\begin{aligned}
& \lambda \chi_{\rho}\left(\Omega_{n}(G, K) ; \lambda\right)=n !|G|^{n}(-1)^{n} Z\left(S_{n}\right)\left[x_{t} \rightarrow \frac{-\lambda}{|G|} \chi_{r}(K ; \lambda)\right]
\end{aligned}
$$

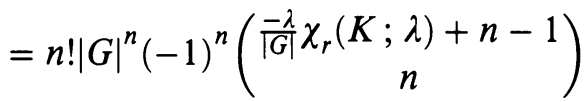

$$
\begin{aligned}
& =n !|G|^{n}\left(\begin{array}{c}
\frac{\lambda}{|G|} \chi_{r}(K ; \lambda) \\
n
\end{array}\right) \\
& =\prod_{i=0}^{n-1}\left(\lambda \chi_{r}(K ; \lambda)-i|G|\right) \text {. }
\end{aligned}
$$

\subsection{Generalized Dowling lattices.}

Definition 2.2.1. We say that a $K$-digraph $\delta$ has the Dowling property if it has at most one connected component with edges labelled by $G$. Let $D_{n}(G, K)$ denote the set of all $K$-digraphs on [ $n$ ] with the Dowling property.

Let $\delta \in D_{n}(G, K)$. Then $\delta$ has a unique (perhaps empty) connected component with edges labelled $G$. We call this connected component the distinguished component of $\delta$ and other connected components are called ordinary components.

For $\delta$ a $K$-digraph, let $\tilde{\delta}$ be the unique $K$-digraph obtained by joining into one connected component all those components with edges labelled $G$. It is easy to see that $\delta \rightarrow \tilde{\delta}$ is a closure operator on $\Omega_{n}(G, K)$ and that $D_{n}(G, K)$ is the set of closed elements. It follows that $D_{n}(G, K)$ is a lattice and moreover a meet-sublattice of $\Omega_{n}(G, K)$. Thus the meet operation in $D_{n}(G, K)$ is described in Definition 2.1.6.

As in the previous subsection we assume that $r$ is a conjugation-invariant rank function on $K$.

Definition 2.2.2. Define a rank function $p=p_{r}$ on $D_{n}(G, K)$ as follows: if $\delta$ has connected components $\delta_{1}, \ldots, \delta_{l}$, where $\delta_{i}$ has $a_{i}$ points and has some loop labelled $H_{i}$, then

$$
\rho(\delta)=\sum_{i} r\left(H_{i}\right)+\left(a_{i}-1\right) r(G)=\rho(\delta)-(n-l) .
$$

Note that $\rho(1)=n r(G)$.

The remainder of this subsection is devoted to computing the characteristic polynomial of $D_{n}(G, K)$ with respect to $\rho$. As in $\S 2.1$ we begin by computing the value of the Möbius function from 0 to 1 . Both of these computations are similar to the corresponding computations for $\Omega_{n}(G, K)$ and therefore we will leave out many of the details. 
Theorem 2.2.3. Let $G, K$, and $D_{n}(G, K)$ be as above. Then

$$
\mu_{D_{n}(G, K)}(0,1)=\prod_{i=0}^{n-1}\left(\mu_{K}(0,1)-i|G|\right) .
$$

Proof. The proof is by induction on $n$, the case $n=1$ being trivial. Assume $n>1$. Let $x$ be the digraph in $D_{n}(G, K)$ with distinguished component consisting of the points $1,2, \ldots, n-1$ and with the loop at $n$ labelled $E$. Let $C_{x}$ denote the set of complements to $x$ in $D_{n}(G, K)$. Using arguments similar to those used in the proof of Theorem 2.1.12, one can show that

$$
C_{x}=\left\{A_{u, n, g}: 1 \leq u \leq n-1, g \in G\right\} \cup\{z\},
$$

where $z$ is the $K$-digraph with distinguished component $\{n\}$ whose only edges outside the distinguished component are loops at each point labelled $E$.

As in the proof of Theorem 2.1.12 one can show that the interval $\left[A_{u, n, g}, 1\right]$ in $D_{n}(G, K)$ is isomorphic to the interval $[0,1]$ in $D_{n-1}(G, K)$. Clearly $A_{u, n, g}$ is an atom in $D_{n}(G, K)$.

The interval $[0, z]$ in $D_{n}(G, K)$ is isomorphic to the interval $[0,1]$ in $K$. It is straightforward to check that the interval $[z, 1]$ in $D_{n}(G, K)$ is isomorphic to the interval $[0,1]$ in $D_{n-1}(G, K)$. Applying Crapo's complementation theorem to $x$ we obtain

$$
\mu_{D_{n}(G, K)}(0,1)=\left\{\mu_{K}(0,1)-(n-1)|G|\right\} \mu_{D_{n-1}(G, K)}(0,1),
$$

which completes the induction step.

Now we are in a position to compute the characteristic polynomial of $D_{n}(G, K)$ in terms of the characteristic polynorinial of $K$.

Theorem 2.2.4. Let $G, K$, and $D_{n}(G, K)$ be as above. Then

$$
\chi_{\rho}\left(D_{n}(G, K) ; \lambda\right)=\prod_{i=0}^{n-1}\left(\chi_{r}(K ; \lambda)-i|G|\right) .
$$

Proof. Let $\delta$ be an element of $D_{n}(G, K)$. Assume that $\delta$ has connected components $\delta_{0}, \delta_{1}, \ldots, \delta_{l}$ of sizes $\alpha, a_{1}, \ldots, a_{l}$ with $\delta_{0}$ the distinguished component. Then the interval $[0, \delta]$ in $D_{n}(G, K)$ is isomorphic to the direct product of the interval $[0,1]$ in $D_{\alpha}(G, K)$ and the intervals $[0,1]$ in $\Omega_{a_{i}}\left(H_{i}, K_{i}\right)$. Here $H_{i}$ is the subgroup labelling the loop on a point of $\delta_{i}$ and $K_{i}$ is the interval $\left[E, H_{i}\right]$ in $K$. So

$$
\begin{aligned}
\mu(0, \delta) \lambda^{\rho(1)-\rho(\delta)}= & \left\{\prod_{i=0}^{\alpha-1}\left(\mu_{K}(0,1)-i|G|\right)\right\} \\
& \cdot\left\{\prod_{j=1}^{l}\left(\mu_{K}\left(E, H_{j}\right) \lambda^{r(G)-r\left(H_{j}\right)}\left(-\left|H_{j}\right|\right)^{a_{j}-1}\left(a_{j}-1\right) !\right)\right\} .
\end{aligned}
$$

We construct all possible $\delta$ in $D_{n}(G, K)$ according to the following procedure: 
Step 1. Choose some set of $\alpha$ points to go in the distinguished component $(0 \leq \alpha \leq n)$. This can be done in $\left(\begin{array}{l}n \\ \alpha\end{array}\right)$ ways.

Step 2. Choose the size of the ordinary components as well as points to go in them. If there are to be $j_{i}$ ordinary components of size $i$ then this can be done in $(n-\alpha) ! / \prod_{i}(i !)^{j_{i}} j_{i}$ ! ways.

Step 3 . For each ordinary component $\delta_{s}$, choose a point $u_{s}$ from $\delta_{s}$, a subgroup $H_{s}$ to label the loop at $u_{s}$, and cosets $H_{u_{s}, v}$ to label the edge from $u_{s}$ to $v$ for all $v \neq u_{s}$ in $\delta_{s}$. These choices can be made in

$$
\sum_{s} \sum_{H_{s} \neq G}\left(\frac{|G|}{\left|H_{s}\right|}\right)^{\left|\delta_{s}\right|-1}
$$

ways.

The choices made in Steps 1-3 are independent and completely determine a $K$-digraph $\delta$ in $D_{n}(G, K)$. Summing equation $(2.2 .5)$ over all these choices we obtain:

$$
\begin{aligned}
& \chi_{\rho}\left(D_{n}(G, K) ; \lambda\right) \\
& =\sum_{\alpha=0}^{n}\left(\begin{array}{l}
n \\
\alpha
\end{array}\right)\left\{\prod_{i=0}^{\alpha-1}\left(\mu_{K}(0,1)-i|G|\right)\right\} \\
& \quad \cdot \sum_{j_{1}, \ldots, j_{n-\alpha}}\left(\frac{(n-\alpha) !}{\prod_{i} i^{j_{i}} j_{i} !}\right) \prod_{i}\left(\sum_{H<G} \mu_{K}(0, H) \lambda^{r(G)-r(H)}|G|^{i-1}\right)^{j_{i}},
\end{aligned}
$$

where the second sum on the right is over sequences $j_{1}, \ldots, j_{n-\alpha}$ such that $\sum i j_{i}=n-\alpha$.

Note that

$$
\begin{aligned}
& \frac{1}{\alpha !} \prod_{i=0}^{\alpha-1}\left(\mu_{K}(0,1)-i|G|\right)=|G|^{\alpha}\left(\begin{array}{c}
\mu_{K}(0,1) /|G| \\
\alpha
\end{array}\right), \\
& \sum_{H<G} \mu_{K}(0, H) \lambda^{r(G)-r(H)}=\chi_{r}(K, \lambda)-\mu_{K}(0,1) .
\end{aligned}
$$

Substituting (2.2.7) and (2.2.8) in (2.2.6) we obtain

$$
\begin{aligned}
\chi_{\rho}\left(D_{n}(G, K) ; \lambda\right)= & n ! \sum_{\alpha=0}^{n}|G|^{\alpha}\left(\begin{array}{c}
\mu_{K}(0,1) /|G| \\
\alpha
\end{array}\right)|G|^{n-\alpha}(-1)^{n-\alpha} \\
& \cdot Z\left(S_{n-\alpha}\right)\left[x_{t} \leftarrow \frac{\left(\chi_{r}(K ; \lambda)-\mu_{K}(0,1)\right)}{|G|}\right] .
\end{aligned}
$$

Using the well-known fact that $Z\left(S_{m}\right)\left[x_{t} \leftarrow X\right]=(-1)^{m}\left(\begin{array}{c}-X \\ m\end{array}\right)$ we obtain

$$
\begin{aligned}
\chi_{\rho}\left(D_{n}(G, K) ; \lambda\right) & =n !|G|^{n} \sum_{\alpha=0}^{n}\left(\begin{array}{c}
\mu_{K}(0,1) /|G| \\
\alpha
\end{array}\right)\left(\begin{array}{c}
\left(\chi_{r}(K ; \lambda)-\mu_{K}(0,1)\right) /|G| \\
n-\alpha
\end{array}\right) \\
& =n !|G|^{n}\left(\begin{array}{c}
\chi_{r}(K ; \lambda) /|G| \\
n
\end{array}\right)
\end{aligned}
$$

and the result follows immediately. 
We end this section with an important example of generalized Dowling lattices. Let $G$ be a finite nontrivial group, i.e., $2 \leq|G|<\infty$. Define the closure $H \rightarrow \bar{H}$ on $L(G)$ by

$$
\bar{H}= \begin{cases}E & \text { if } H=E, \\ G & \text { otherwise. }\end{cases}
$$

Every subgroup closes to $G$ except the trivial subgroup $E$ which is itself closed. Thus

$$
K=\left.\right|_{E} ^{G}
$$

and so $\mu_{K}(0,1)=-1$ and $\chi_{r}(K ; \lambda)=\lambda-1$. For $r$ we take the usual rank function. In this case $D_{n}(G, K)$ is isomorphic to the Dowling lattice $D_{n}(G)$ of rank $n$ associated to the finite group $G$ (see [5]). Moreover the rank function $\rho$ is the usual rank function on $D_{n}(G)$. Theorem 2.2.4 reduces to the familiar formula

$$
\chi\left(D_{n}(G) ; \lambda\right)=\prod_{i=0}^{n-1}(\lambda-(1+i|G|))
$$

obtained by Dowling using the fact that $D_{n}(G)$ is a supersolvable geometry. The theory of supersolvable geometries gives an explanation for why the roots of $\chi\left(D_{n}(G) ; \lambda\right)$ are nonnegative integers. It is easy to see that the generalized Dowling lattices are not supersolvable except in the Dowling lattice case. Nonetheless the roots of their characteristic polynomials, with respect to the variable $\chi_{r}(K ; \lambda)$, are nonnegative integers.

\section{REMOVING THE THICK DIAGONAL WITH RESPECT TO A REPRESENTATION}

In this section we consider the example which originally led us to define the generalized Dowling lattices. Throughout this section we assume that $\varphi: G \rightarrow$ $\mathrm{GL}(V)$ is a finite-dimensional faithful (complex) representation of $G$ which does not contain the trivial representation. For each subgroup $H$ of $G$ define $V_{H}$ by

$$
V_{H}=\{v \in V: \varphi(h) v=v \text { for all } h \in H\} .
$$

If $V_{H}=V_{L}$ for subgroups $H$ and $L$ then $V_{\langle H, L\rangle}=V_{H}=V_{L}$, where $\langle H, L\rangle$ is the subgroup generated by $H$ and $L$. So the following definition makes sense.

Definition 3.1.1. Let $H$ be a subgroup of $G$. Define $\bar{H}$ to be the maximal subgroup of $G$ satisfying $V_{\bar{H}}=V_{H}$.

It is easy to see that the map $H \rightarrow \bar{H}$ is a closure on $L(G) \quad(\bar{E}=E$ since $\varphi$ is faithful). Let $K(\varphi)$ denote the lattice of closed subgroups of $G$. Note that

$$
\overline{g^{-1} H g}=g^{-1} \bar{H} g \quad \text { for } g \in G, H \in L(G) .
$$

So $H \rightarrow \bar{H}$ is a conjugation-invariant closure. 
Let $\mathscr{V}$ denote the set of subspaces $\mathscr{U}$ in $V$ such that $\mathscr{U}=V_{H}$ for some subgroup $H$. Then $\mathscr{V}$, ordered by inclusion, is the lattice dual to $K(\varphi)$. The maps

$$
H \rightarrow V_{H} \text { and } \mathscr{U} \rightarrow G_{\mathscr{U}}=\{g \in G: \varphi(g) \mathscr{U} \subseteq \mathscr{U}\}
$$

give a Galois connection in the sense of Rota [15]. The condition that $\varphi$ not contain the trivial representation implies that $V_{G}=0$. So the maximal element of $\mathscr{V}$ is $V=V_{E}$ and the minimal element is $0=V_{G}$.

Let $n$ be a positive integer and let $i, j$ satisfy $1 \leq i \leq j \leq n$. For $g \in G$, let $\mathscr{H}(i, j, g)$ denote the subspace of $V^{n}$ given by

$$
\mathscr{H}(i, j, g)=\left\{\left(v_{1}, \ldots, v_{n}\right): v_{j}=\varphi(g) v_{i}\right\} .
$$

For $i \neq j, \mathscr{H}(i, j, g)$ has codimension $d=\operatorname{dim} V$ in $V^{n}$. For $i=j$ the codimension of $\mathscr{H}(i, j, g)$ is $d-\operatorname{dim}\left(V_{\langle g\rangle}\right)$, where $\langle g\rangle$ is the subgroup of $G$ generated by $g$. In particular, $\mathscr{H}(i, i, e)=V^{n}$ for all $i$, and $\mathscr{H}(i, i, g)$ is a proper subspace of $V^{n}$ for $g \neq e$ (since $\varphi$ is faithful).

Definition 3.1.2. Let $n$ be a positive integer and let $\varphi: G \rightarrow \mathrm{GL}(V)$ be a faithful representation of $G$ which does not contain the trivial representation. Define $\mathscr{S}_{n}(\varphi)$ to be the set of subspaces

$$
\begin{aligned}
\mathscr{S}_{n}(\varphi)= & \{\mathscr{H}(i, j, g): 1 \leq i<j \leq n, g \in G\} \\
& \cup\{\mathscr{H}(i, i, g): 1 \leq i \leq n, g \in G \backslash\{e\}\} .
\end{aligned}
$$

Let $L_{n}(\varphi)$ be the lattice of intersections of these subspaces ordered by reverse inclusion and let $\Lambda_{n}(\varphi)$ be the subset of $V^{n}$ obtained by removing the union of the subspaces in $\mathscr{S}_{n}(\varphi)$, i.e.,

$$
\Lambda_{n}(\varphi)=V^{n} \backslash\left(\bigcup_{\mathscr{L} \in \mathscr{S}_{n}(\varphi)} \mathscr{H}\right) .
$$

Later in this paper we will compute the homology of $\Lambda_{n}(\varphi)$ as a topological space. To do this we instead compute the ordinary poset homology of $L_{n}(\varphi)$ and then invoke results of Goresky and Macpherson which describe the homology of $\Lambda_{n}(\varphi)$ in terms of the homology of $L_{n}(\varphi)$. To compute the homology of $L_{n}(\varphi)$ we need a combinatorial description of $L_{n}(\varphi)$. We will prove that

$$
L_{n}(\varphi) \cong D_{n}(G, K(\varphi))
$$

and so the generalized Dowling lattices give such a description.

It is important to identify the atoms of $L_{n}(\varphi)$. One might think that these are exactly the subspaces $\mathscr{H}(i, j, g)$. However that is not quite correct because

$$
\mathscr{H}(i, i, j) \subset \mathscr{H}(i, i, h) \text { if } \overline{\langle h\rangle} \subseteq \overline{\langle g\rangle} .
$$

So not all the subspaces $\mathscr{H}(i, j, g)$ are atoms in $L_{n}(\varphi)$ and not all the subspaces $\mathscr{H}(i, j, g)$ are distinct. We have $\mathscr{H}(i, i, g)=\mathscr{H}(i, i, h)$ whenever 
$\overline{\langle g\rangle}=\overline{\langle h\rangle}$. The set of atoms $\mathscr{A}_{n}(\varphi)$ in $L_{n}(\varphi)$ is given by

$$
\begin{aligned}
\mathscr{A}_{n}(\varphi)= & \{\mathscr{H}(i, j, g): 1 \leq i<j \leq n, g \in G\} \\
& \cup\{\mathscr{H}(i, i, g): 1 \leq i \leq n, \overline{\langle g\rangle} \text { an atom in } K(\varphi)\} .
\end{aligned}
$$

In the latter set we need only one $g$ for each atom in $K(\varphi)$ so a better way to denote the second set would be the set of $\mathscr{H}(i, i, \Gamma)$ such that $\Gamma$ is an atom in $K(\varphi)$.

Next we define maps $u, w$ which we will eventually prove to be isomorphisms between $L_{n}(\varphi)$ and $D_{n}(G, K(\varphi))$. For the moment we only assume that $u$ maps $L_{n}(\varphi)$ to the set of $G$-labelled digraphs with the Dowling property and that $w$ maps $D_{n}(G, K(\varphi))$ to $V^{n}$.

Definition 3.1.4. Let $\mathscr{I}$ be an element of $L_{n}(\varphi)$. Define the $G$-labelled digraph $u(\mathscr{F})$ with $n$ points and edge labels $H_{i j}$ by

$$
H_{i j}=\left\{g \in G: v_{j}=\varphi(g) v_{i} \text { for all }\left(v_{1}, \ldots, v_{n}\right) \in \mathscr{I}\right\}
$$

(as usual, if $H_{i j}=\varnothing$ then there is no edge in $u(\mathscr{I})$ from $i$ to $j$ ).

Because $\varphi$ is a representation it follows easily that

(1) If $(i, j, k) \in[n]^{3}$, then $H_{j k} H_{i j}=H_{i k}$.

(2) For all $i \in[n], e \in H_{i i}$. In particular, $H_{i i}$ is nonempty.

From (1) and (2) it follows that $H_{i i}$ is a subgroup of $G$ and $H_{i j}$ is a right coset of $H_{i i}$ (see the proof of Proposition 2.1.3). Since $\varphi$ does not contain the trivial representation we have

(3) $H_{i i}=G$ iff $v_{i}=0$ for all $\left(v_{1}, \ldots, v_{n}\right) \in \mathscr{I}$.

From (3) it follows immediately that if $H_{i i}=H_{j j}=G$, then $H_{i j}=G$. So $u(\mathscr{I})$ has the Dowling property.

For each $i<j$ and each $g \in G$ let $A_{i, j, g}$ denote the atom in $D_{n}(G, K(\varphi))$ with loops at every point labelled $E$ and two other edges-one from $i$ to $j$ labelled $\{g\}$ and one from $j$ to $i$ labelled $\left\{g^{-1}\right\}$. For each atom $\Gamma$ in $K(\varphi)$ let $A_{i i \Gamma}$ denote the atom in $D_{n}(G, K(\varphi))$ which has no other edges except the loops at every point. All the loops are labelled $E$ except for the loop at $i$ which is labelled $\Gamma$.

Lemma 3.1.5. For $1 \leq i<j \leq n$ and $g \in G$ we have $u\left(\mathscr{H}_{i, j, g}\right)=A_{i, j, g}$ and for $1 \leq i \leq n$ and $\Gamma$ an atom in $K(\varphi)$ we have $u\left(\mathscr{H}_{i, i, \Gamma}\right)=A_{i, i, \Gamma}$.

The proof of Lemma 3.1.5 is easy and is left to the reader. Lemma 3.1.5 asserts that $u$ is a bijection between the atoms of $L_{n}(\varphi)$ and the atoms of $D_{n}(G, K(\varphi))$.

Definition 3.1.6. Define $w: D_{n}(G, K(\varphi)) \rightarrow V^{n}$ in the following way. If $\delta$ is a digraph with edge labels $H_{i j}$, then

$$
w(\delta)=\left\{\left(v_{1}, \ldots, v_{n}\right): v_{j}=\varphi(g) v_{i} \text { for all } g \in H_{i j}, \text { all } i, j\right\} .
$$


Lemma 3.1.7. Let $\delta \in D_{n}(G, K(\varphi))$. Then $u w \delta=\delta$.

Proof. Let $H_{i j}$ and $K_{i j}$ be the edge labels on $\delta$ and $u w \delta$ respectively. It is easy to see that $H_{i j} \subseteq K_{i j}$ and so we need to prove the reverse inclusions. Clearly it is enough to show that $H_{i i} \supseteq K_{i i}$ for all $i$.

Let $g \in K_{i i}$ and let $v_{i} \in \mathscr{V}_{H_{i i}}$. Define a vector $\mathbf{v}=\left(v_{1}, \ldots, v_{n}\right) \in V^{n}$ according to the following rules.

Rule 1. If $r$ and $i$ are not in the same connected component of $\delta$ then $v_{r}=0$.

Rule 2. If $r$ and $i$ are in the same connected component choose $\gamma_{i r} \in H_{i r}$ and set $v_{r}=\varphi\left(\gamma_{i r}\right) v_{i}$.

We claim that $\mathbf{v} \in w(\delta)$. To prove this we need to show that $v_{s}=\varphi(l) v_{r}$ whenever $l \in H_{r s}$. This is obvious unless both $r$ and $s$ are in the same connected component as $i$. In that case, $H_{r s}=\gamma_{i s} H_{i i} \gamma_{i r}^{-1}$ so $l=\gamma_{i s} h \gamma_{i r}^{-1}$ for some $h \in H_{i i}$. Thus

$$
\begin{aligned}
\varphi(l) v_{r} & =\varphi\left(\gamma_{i s}\right) \varphi(h) \varphi\left(\gamma_{i r}^{-1}\right) v_{r} \\
& =\varphi\left(\gamma_{i s}\right) \varphi(h) v_{i}=\varphi\left(\gamma_{i s}\right) v_{i}=v_{s}
\end{aligned}
$$

which proves $\mathbf{v} \in w(\delta)$.

Now return to $g \in K_{i i}$. By the definition of $u$ we have $\varphi(g) v_{i}=v_{i}$. So $\varphi(g)$ fixes every $v_{i}$ in $\mathscr{V}_{H_{i i}}$ or equivalently $\mathscr{V}_{H_{i i}} \subseteq \mathscr{V}_{K_{i i}}$. Since $H_{i i}$ is closed in $K(\varphi)$ we have $K_{i i} \subseteq H_{i i}$ which completes the proof.

Lemma 3.1.8. Let $\mathscr{I} \in L_{n}(\varphi)$. Then $w u \mathscr{I}=\mathscr{I}$.

Proof. It is immediate that $\mathscr{I} \subseteq w u \mathscr{I}$ and so we show containment in the other direction. Suppose that

$$
\mathscr{I}=\bigcap_{\mathscr{H} \in S} \mathscr{H}
$$

where $S$ is some nonempty subset of the atoms of $L_{n}(\varphi)$. Let $\mathscr{H}_{i j g}$ be an atom in $S$ where $i<j$ and $g \in G$. Note that $u$ is an order-preserving map, i.e., if $\mathscr{J} \subseteq \mathscr{I}$ then $u(\mathscr{J}) \geq u(\mathscr{I})$ (recall that the order on $L_{n}(\varphi)$ is reverse inclusion). So $u(\mathscr{I}) \leq u\left(\mathscr{H}_{i j g}\right)=A_{i, j, g}$. Hence $g \in H_{i j}$, where the $H_{u v}$ are the edge-labels on $u(\mathscr{I})$. So if $\mathbf{v}=\left(v_{1}, \ldots, v_{n}\right)$ is in $w u(\mathscr{I})$, then $v_{j}=\varphi(g) v_{i}$ hence $\mathbf{v} \in \mathscr{H}_{i j g}$. By a similar argument $w u \mathcal{F} \subseteq \mathscr{H}_{i i \Gamma}$ is in $S$. Thus $w u \mathscr{I} \subseteq \mathscr{I}$ which proves Lemma 3.1.8.

We can now state the main result of this section.

Theorem 3.1.9. For all $n$ and $\varphi$ we have $L_{n}(\varphi) \cong D_{n}(G, K(\varphi))$. Moreover the maps $u$ and $w$ defined above are isomorphisms with $w=u^{-1}$.

Proof. We have proved this theorem in Lemmas 3.1.7 and 3.1.8 modulo one technical point. We have not shown that the image of $u$ is contained in $D_{n}(G, K(\varphi))$, i.e., that the subgroup labelling each loop in $u(\mathscr{F})$ is in $K(\varphi)$. This argument is similar to the proof that $K_{i i}=H_{i i}$ which was done in Lemma 3.1.7 and is left to the reader. 
Let $G$ be a finite group. It is natural to ask whether there exists a representation $\varphi$ of $G$ such that the generalized Dowling lattices $D_{n}(G, K(\varphi))$ are the ordinary Dowling lattices $D_{n}(G)$. This is equivalent to saying that $K(\varphi)$ consists only of $E$ and $G$, i.e., no nontrivial subgroup of $G$ has a fixed vector under $\varphi$. One example of this is when $G$ is the cyclic group $\mathscr{C}_{m}=\left\langle\sigma_{m}\right\rangle$ and $\varphi$ is the 1-dimensional representation $\varphi\left(\delta_{m}\right)=e^{2 \pi i / m}$. Zassenhaus classified all groups having a representation with this property. His classification can be found in Passman [13]. My thanks to Peter Cameron for bringing this to my attention.

\section{THE HOMOLOGY OF $D_{n}(G, K)$}

In this section we compute the homology of $\Omega_{n}(G, K)$ and the homology of $D_{n}(G, K)$, both in terms of the homology of $K$. We will go on to give a second computation of $H_{*}\left(D_{n}(G, K)\right)$. This second computation will express $H_{*}\left(D_{n}(G, K)\right)$ in quite a different form. The purpose for this second computation is that we can use it to determine the representation of the automorphism group of $D_{n}(G, K)$ on the homology groups $H_{*}\left(D_{n}(G, K)\right)$.

4.1. Homology groups of posets. Let $(P, \leq)$ be a finite poset with a unique maximal element 1 and a unique minimal element 0 . For each nonnegative integer $r$ let $c_{r}(P)$ be the set of chains in $P$ given by

$$
c_{r}(P)=\left\{0<x_{1}<x_{2}<\cdots<x_{r}<1: x_{i} \in P\right\} .
$$

Let $C_{r}(P)$ denote the complex vector space with basis $c_{r}(P)$. Define $\partial_{r}: C_{r}(P)$ $\rightarrow C_{r-1}(P)$ by

$$
\partial_{r}\left(0<x_{1}<\cdots<x_{r}<1\right)=\sum_{i=1}^{r}(-1)^{i-1}\left(0<x_{1}<\cdots<\hat{x}_{i}<\cdots<x_{r}<1\right) .
$$

It is easy to check that $\partial_{r} \circ \partial_{r+1}=0$. Define the $r$ th homology group of $P$, $H_{r}(P)$, by

$$
H_{r}(P)=\operatorname{ker} \partial_{r} / \operatorname{im} \partial_{r+1} .
$$

We do allow $0<1$ as an element of $C_{0}(P)$ so $C_{0}(P)$ has dimension 1 .

We will encounter the situation where $P$ is completely trivial, i.e., $0=1$. In this case we define $H_{r}(P)$ by

$$
H_{r}(P)= \begin{cases}\mathbb{C} & \text { if } r=-1 \\ 0 & \text { if } r \neq-1\end{cases}
$$

Note that the chains in $c_{r}(P)$ must include 0 and 1 . Many authors would define $H_{r}(P)$ differently, working with $P^{\prime}=P \backslash\{0,1\}$ and allowing $r$-chains to be arbitrary.

A fundamental result about homology groups of posets in the following Euler characteristic equation (see Rota [15] or Stanley [16]). 
Theorem 4.1.1. Let $P$ be a finite poset with unique maximal and minimal elements 1 and 0 . Then

$$
\sum_{r}(-1)^{r} \operatorname{dim}\left(H_{r}(P)\right)=\mu_{P}(0,1) .
$$

Our computation will be based on the following theorem due to Björner and Walker [2] which one can think of as a homological version of Crapo's complementation theorem.

Theorem 4.1.2 (Björner and Walker). Let $L$ be a finite lattice and let $x \in$ $L \backslash\{\hat{0}, \hat{1}\}$. Assume that the complements of $x$ form an antichain. Then

$$
H_{*}(L) \cong \bigoplus_{y} H_{*}([\hat{0}, y]) \otimes H_{*}([y, \hat{1}])
$$

where the sum is over complements $y$ to $x$.

We will apply Theorem 4.1.2 to compute the homology groups of $\Omega_{n}(G, K)$ and $D_{n}(G, K)$. For $L$ any poset define Poincaré series for $L, P(L ; t)$, to be the polynomial

$$
P(L ; t)=\sum_{i} \operatorname{dim}\left(H_{i}(L)\right) t^{i}
$$

Theorem 4.1.3. $H_{*}\left(\Omega_{n}(G, K)\right)$ is isomorphic to a direct sum of $|G|^{n-1}$ copies of $H_{*}\left(\Pi_{n}\right) \otimes H_{*}(K)$, where $\Pi_{n}$ denotes the lattice of partitions of $\{1,2, \ldots, n\}$. In terms of Poincaré series,

$$
P\left(\Omega_{n}(G, K) ; t\right)=(n-1) !(t|G|)^{n-1} P(K ; t) .
$$

Proof. Let $x$ be the $K$-digraph whose only edges consist of a loop at every point labelled $G$. Let $C(x)$ denote the set of complements to $x$. It is straightforward to check that $y \in C(x)$ iff $y$ is connected and every loop of $y$ is labelled $E$. In particular, $|C(x)|=|G|^{n-1}$.

It is also straightforward to check that if $y \in C(x)$ then

$$
[\hat{0}, y] \cong \Pi_{n} \text { and }[y, \hat{1}] \cong K \text {. }
$$

The proof follows immediately from Theorem 4.1.2.

The next result gives the Poincaré series for $D_{n}(G, K)$. This Poincaré series determines the dimension of the homology of $D_{n}(G, K)$ in each degree. In $\S \S 4.2-4.4$ we will give a more explicit computation of $H_{*}\left(D_{n}(G, K)\right)$. The Poincare series that we compute here will be crucial for that upcoming work.

Theorem 4.1.4. The Poincaré series of $D_{n}(G, K)$ is given by

$$
P\left(D_{n}(G, K) ; t\right)=\prod_{i=0}^{n-1}(P(K ; t)+i|G| t) .
$$

Proof. We will prove this by induction on $n$, the case $n=1$ being trivial. For $n>1$ we will use Theorem 4.1 .2 with $x$ being the $K$-digraph whose only edges 
are loops with every loop labelled $E$ except the loop at $n$ which is labelled $G$. Let $y$ be a complement to $x$ and let $S$ be the distinguished component of $x$. Note that $n$ is not in $S$ so $S \subseteq(\mathbf{n}-\mathbf{1})$. Let $\bar{S}$ be $\mathbf{n} \backslash S$. Since $x \vee y=\hat{1}$, the subgraph $\bar{y}$ of $y$ induced by the points in $\bar{S}$ must be connected. Since $x \wedge y=\hat{0}$, every loop in $\bar{y}$ must be labelled $E$.

It is easy to see that the interval $[0, y]$ is isomorphic to $D_{|S|}(G, K) \times \Pi_{n-|S|}$ and that the interval $[y, 1]$ is isomorphic to $K$. Applying the Björner-Walker theorem we have that

$$
P\left(D_{n}(G, K) ; t\right)=\sum_{u=0}^{n-1}\left(\begin{array}{c}
n-1 \\
u
\end{array}\right) t^{u} u !|G|^{u} P(K ; t) P\left(D_{n-u-1}(G, K) ; t\right) .
$$

In this sum $u$ denotes $|\bar{S} \backslash\{n\}|$ so that the factors on the right-hand side are accounted for as follows:

(1) $\left(\begin{array}{c}n-1 \\ u\end{array}\right)$ is the number of choices of $\bar{S} \backslash\{n\}$ having size $u$.

(2) $|G|^{u}$ is the number of choices for $\bar{y}$, the connected component of $y$ containing $n$.

(3) $u$ ! is the dimension of $H_{*}\left(\Pi_{u+1}\right)$, so

$$
u ! t^{u} P\left(D_{n-u-1}(G, K) ; t\right)
$$

is the Poincare series for the homology of $[0, y]$.

(4) $P(K ; t)$ is the Poincare series for the homology of $[y, 1]$.

By our induction hypothesis we have

$$
P\left(D_{n-u-1}(G, K) ; t\right)=\prod_{i=0}^{n-u-2}(P(K ; t)+i|G| t) .
$$

Substituting in (4.1.5) we have

$$
\begin{aligned}
P\left(D_{n}(G, K) ; t\right) & =\sum_{u=0}^{n-1}\left(\begin{array}{c}
n-1 \\
u
\end{array}\right) t^{u} u !|G|^{u} P(K ; t) \prod_{i=0}^{n-u-2}(P(K ; t)+i|G| t) \\
& =\sum_{u=0}^{n-1}\left\{\prod_{j=1}^{u}(n-j) t|G|\right\} P(K ; t) \prod_{i=0}^{n-u-2}(P(K ; t)+i|G| t) .
\end{aligned}
$$

To see that the right-hand side of (4.1.6) agrees with the right-hand side of Theorem 4.1.4 expand the latter as

$$
\sum_{S \subseteq\{0,1, \ldots, n-1\}} P(K ; t)^{n-|S|} \prod_{i \in S} i|G| t=\sum_{u=0}^{n-1} \sum_{S^{\prime}} P(K ; t)^{n-\left|S^{\prime}\right|} \prod_{i \in S^{\prime}} i|G| t,
$$

where the sum is over sets $S^{\prime}$ such that $n-u, n-u+1, \ldots, n-1$ are in $S$ but $n-u-1$ is not in $S^{\prime}$. It is straightforward to see that the last expression is equal to the right-hand side of (4.1.6).

4.2. The filtration of $H_{*}(P)$ with respect to an order ideal of $P$. The purpose of this subsection is to describe a combinatorial method for constructing a filtration 
of the order complex associated to a partially ordered set. Let $P$ be a finite poset with unique minimal and maximal elements 0 and 1 . Let $\mathscr{I}$ be an order ideal in $P$ which satisfies the Jordan-Hölder property. Thus we can split $\mathscr{I}$ as a disjoint union of sets $\mathscr{I}_{i}$ where $x \in \mathscr{I}_{i}$ if and only if every maximal $0-x$ chain has length $i$. We call $i$ the rank of $x$ and write $\operatorname{gr}(x)=i$.

Let $\Gamma: 0=x_{0}<x_{1}<\cdots<x_{r}<1$ be an $r$-chain in $P$. Define the pivot of $\Gamma, p(\Gamma ; \mathscr{I})$, to be the maximal $x_{j}$ such that $x_{j} \in \mathscr{I}$. Define the weight of $\Gamma$ to be the rank of its pivot and define $\mathscr{C}_{r, i}$ to be the linear span of all $r$-chains with weight $i$.

Let $d_{r}: \mathscr{C}_{r} \rightarrow \mathscr{C}_{r-1, *}$ be the usual boundary map for computing the homology of $P$. It is easy to see that

$$
d_{r}: \mathscr{C}_{r, i} \rightarrow \bigoplus_{j \leq i} \mathscr{C}_{r-1, j}
$$

So we have a filtered complex.

As in $\S 4.1$, let $d_{r}^{+}$be that part of the boundary which preserves weight, i.e.,

$$
d_{r}^{+}: \mathscr{C}_{r, i} \rightarrow \mathscr{C}_{r-1, i} \text { for all } i .
$$

It is easy to describe the map $d_{r}^{+}$combinatorially. Let $\Gamma$ be an $r$-chain,

$$
\Gamma: 0=x_{0}<x_{1}<\cdots<x_{j-1}<p(\Gamma)<x_{j+1}<\cdots<x_{r}<1,
$$

where $p(\Gamma)=p(\Gamma ; \mathscr{I})$ is the pivot. Then

$$
\begin{aligned}
\partial_{r}^{+}(\Gamma)= & \sum_{i=1}^{j-1}(-1)^{i-1}\left(0<x_{1}<\cdots<\hat{x}_{i}<\cdots<x_{j-1}<p(\Gamma)<\cdots<1\right) \\
& +\sum_{i=j+1}^{r}(-1)^{i-1}\left(0<x_{1}<\cdots<p(\Gamma)<x_{j+1}<\cdots<\hat{x}_{i}<\cdots<x_{r}<1\right) .
\end{aligned}
$$

From (4.2.1) it is easy to see that $E^{1}=H_{*}\left(\mathscr{C}, d^{+}\right)$is given by

$$
E^{1}=\bigoplus_{\alpha \in \mathcal{I}} H_{*}([0, \alpha]) \otimes H_{*}\left([\alpha, 1]_{\mathscr{J}}\right),
$$

where $H_{*}([0, \alpha])$ is the poset homology of the interval $[0, \alpha]$ and where $H_{*}\left([\alpha, 1]_{\mathcal{F}}\right)$ is the poset homology of

$$
[\alpha, 1]_{\mathscr{I}} \equiv[\alpha, 1] \backslash([\alpha, 1] \cap \mathscr{F}) .
$$

In $\S 4.4$ we will make use of such a spectral sequence to compute $H_{*}\left(D_{n}(G, K)\right)$. In that case we will have the additional information that every interval of $\mathscr{I}$ is Cohen-Macaulay. Because of this Cohen-Macaulay property, the differential $d^{1}$ on $E^{1}$ will have a particularly simple form. 


\subsection{Elementary digraphs.}

Definition 4.3.1. Let $\delta \in D_{n}(G, K)$. We say $\delta$ is elementary if every loop of $\delta$ is labelled by the trivial subgroup $E$. We let $\mathscr{I}=\mathscr{I}_{n}(G)$ denote the set of elementary digraphs in $D_{n}(G, K)$ (note that $\mathscr{I}$ does not depend on $K$ ).

Note that $\mathscr{I}$ is an order ideal in $D_{n}(G, K)$ and that if $\delta \in \mathscr{I}$ then the interval $[0, \delta]$ in $D_{n}(G, K)$ is isomorphic to the interval $[0, \pi(\delta)]$ in $\Pi_{n}$, where $\pi(\delta)$ is the partition of $n$ whose blocks are the connected components of $\delta$.

Definition 4.3.2. Let $\alpha, \beta$ be digraphs in $D_{n}(G, K)$ with $\alpha<\beta$ and $\alpha$ elementary. We say $\beta$ is nondefective over $\alpha$ provided that whenever two components of $\alpha$ are contained in the same component $C$ of $\beta$, that component $C$ must be the distinguished component. Otherwise we say $\beta$ is defective over $\alpha$.

Lemma 4.3.3. Suppose $\beta$ is defective over $\alpha$ and $\beta$ is not elementary. Then there is a unique maximal digraph $\kappa_{\alpha}(\beta)$ satisfying.

(1) $\alpha<\kappa_{\alpha}(\beta)<\beta$.

(2) $\kappa_{\alpha}(\beta)$ is nondefective over $\alpha$.

Proof. Define $\kappa_{\alpha}(\beta)$ to be the digraph obtained from $\beta$ by removing all edges $\varepsilon$ of $\beta$ which satisfy:

(i) $\varepsilon$ is not an edge of $\alpha$.

(ii) The endpoints of $\varepsilon$ lie outside the distinguished component of $\beta$.

It is straightforward to check that this $\kappa_{\alpha}(\beta)$ is the unique maximal digraph satisfying (1) and (2).

Let $\alpha$ be an element of $\mathcal{I}$. A nondefective chain over $\alpha$ is a chain

$$
\alpha<\beta_{i}<\cdots<\beta_{l}<1
$$

in the interval $[\alpha, 1]$ such that each $\beta_{i}$ is nondefective over $\alpha$ and such that no $\beta_{i}$ is elementary. Let $\mathrm{ND}_{*}(\alpha)$ denote the space of nondefective chains over $\alpha$ and let $\partial_{\alpha}$ denote the differential for the poset $[\alpha, 1]$.

Lemma 4.3.4. Let $\alpha$ be an elementary digraph with $r$ connected components. Then the complex $\left(\mathrm{ND}_{*}(\alpha), \partial_{\alpha}\right)$ is isomorphic to the complex $\left(C_{*}\left(K^{r}\right), \partial\right)$, where $K^{r}$ denotes the direct sum of $r$ copies of $K$. In particular,

$$
H_{*}\left(\mathrm{ND}(\alpha), \partial_{\alpha}\right) \cong\left(H_{*}(K)\right)^{\otimes r} .
$$

Proof. Let $X_{1}, \ldots, X_{r}$ be the connected components of $\alpha$ and let $x_{i}$ be a fixed element from $X_{i}$. If $\beta$ is nondefective over $\alpha$ then $\beta$ is determined uniquely by the sequence $\left(h_{1}(\beta), h_{2}(\beta), \ldots, h_{r}(\beta)\right) \in K^{r}$, where $h_{i}(\beta)$ is the subgroup labelling the loop at $x_{i}$. It is straightforward to check that the correspondence

$$
B \rightarrow\left(h_{1}(\beta), \ldots, h_{r}(\beta)\right)
$$

extends to an isomorphism of chain complexes. 
4.4. The homology of $D_{n}(G, K)$. We will now recompute $H_{*}\left(D_{n}(G, K)\right)$ using a method which yields more information than Theorem 4.1.4. This additional information will be crucial in $\S 5$ when we compute the character of $G$ wr $S_{n}$ on $H_{*}\left(D_{n}(G, K)\right)$. We will compute $H_{*}\left(D_{n}(G, K)\right)$ using the spectral sequence $\left(E^{s}, d^{s}\right)$ described in $\S 4.2$ with respect to the order ideal $\mathscr{I}=\mathscr{I}_{n}(G)$ of elementary digraphs. Recall from (4.2.1) that the differential $\partial_{*}^{+}=\partial_{*}^{0}$ applied to a shain $\Gamma$ agrees with the usual differential in $C_{*}\left(D_{n}(G, K)\right)$ except that the term where $p(\Gamma)$ is removed from $\Gamma$ does not appear. Thus

$$
\left(E_{*}^{0}, d^{0}\right) \cong \bigoplus_{\alpha \in \mathcal{I}}\left(E_{*}^{0}(\alpha), d^{0}\right),
$$

where the above isomorphism is an isomorphism of complexes and where $E_{*}^{0}(\alpha)$ is the subcomplex of $E_{*}^{0}$ spanned by all chains with pivot $\alpha$. For each $\alpha$ we will compute the homology of $\left(E_{*}^{0}(\alpha), d^{0}\right)$ using a second spectral sequence.

Fix an elementary digraph $\alpha$ and let $r$ denote the number of connected components of $\alpha$. For each chain $\Gamma$ in $E_{*}^{0}(\alpha)$ let $B(\Gamma)$ be the subchain from $\beta(\Gamma)$ to 1 , where $\beta(\Gamma)$ is the minimal element of $\Gamma$ which is defective over $\alpha$ (define $B(\Gamma)=\varnothing$ if every element of $\Gamma$ is nondefective over $\alpha$ ). Also define $b(\Gamma)$ to be the size of $B(\Gamma)$.

For $b_{0}$ a nonnegative integer let $E_{*}^{0}\left(\alpha ; b_{0}\right)$ denote the span of all chains $\Gamma$ in $E_{*}^{0}(\alpha)$ with $b(\Gamma)=b_{0}$. It is easy to see that

$$
d^{0}\left(E_{*}^{0}\left(\alpha ; b_{0}\right)\right) \subseteq E_{*}^{0}\left(\alpha ; b_{0}\right) \oplus E_{*}^{0}\left(\alpha ; b_{0}-1\right) .
$$

So $b(\Gamma)$ defines a filtration of $\left(E_{*}^{0}(\alpha), d\right)$. Let $\left(F_{*}^{s}, \delta^{s}\right)$ be the associated spectral sequence. We will now compute the terms in this spectral sequence.

It is easy to describe the differential $\delta^{0}$ explicitly. Let $\Gamma$ be a chain in $E_{*}^{0}\left(\alpha ; b_{0}\right)$,

$$
\begin{aligned}
\Gamma: 0 & =\alpha_{0}<\cdots<\alpha_{i-1}<p(\Gamma)<\alpha_{i+1}<\cdots<\alpha_{j} \\
& <\beta(\Gamma)<\beta_{2}<\cdots<\beta_{b_{0}}<1 .
\end{aligned}
$$

Then

$$
\begin{aligned}
\delta^{0}(\Gamma)=\sum_{\substack{s=1 \\
s \neq i}}^{j}(-1)^{s-1}\left(0<\alpha_{1}<\cdots\right. & <\hat{\alpha}_{s}<\cdots<\alpha_{j} \\
& \left.<\beta(\Gamma)<\cdots<\beta_{b_{0}}<1\right) .
\end{aligned}
$$

By (4.4.1) we see that $\left(F^{0}, \delta^{0}\right)$ splits as a direct sum,

$$
\left(F_{*}^{0}, \delta^{0}\right)=\left(E_{*}^{0}(\alpha ; 0), d^{0}\right) \oplus \bigoplus_{\beta_{1}}\left(F_{*}^{0}\left(\beta_{1}\right), \delta^{0}\right),
$$

where the last sum is over $\beta_{1}$ defective over $\alpha, \beta_{1} \notin \mathscr{I} . F_{*}^{0}\left(\beta_{1}\right)$ is the span of all chains $\Gamma$ with $\beta(\Gamma)=\beta_{1}$. 
Consider the complex $\left(F_{*}^{0}\left(\beta_{1}\right), \delta^{0}\right)$. Since $\alpha<\beta_{1}$ and $\beta_{1}$ is defective over $\alpha$ we have $\alpha<\kappa_{\alpha}\left(\beta_{1}\right)<\beta_{1}$. Let $I\left(\alpha ; \beta_{1}\right)$ be the poset consisting of the interval in $D_{n}(G, K)$ from $\alpha$ to $\kappa_{\alpha}\left(\beta_{1}\right)$ together with an additional maximal element $M$.

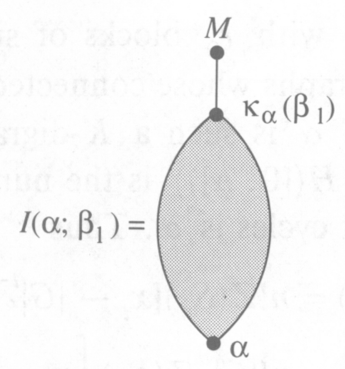

By the usual contraction argument $H_{*}\left(I\left(\alpha ; \beta_{1}\right)\right)=0$. Observe that the complex $\left(F_{*}^{0}\left(\beta_{1}\right), \delta^{0}\right)$ is isomorphic to the tensor product

$$
\left(F_{*}^{0}\left(\beta_{1}\right), \delta^{0}\right) \cong([0, \alpha], \partial) \otimes\left(I\left(\alpha ; \beta_{1}\right), \partial\right),
$$

where both differentials $\partial$ on the right-hand side are the usual poset differentials. It follows immediately from (4.4.1) that an explicit isomorphism $\varphi$ to establish (4.4.2) is given by

$$
\begin{aligned}
\varphi\left(0<\alpha_{1}<\cdots<\alpha_{i-1}<\alpha<\alpha_{i+1}<\cdots<\alpha_{m}<\beta_{1}<\cdots<\beta_{b_{0}}<1\right) \\
=\left(0<\alpha_{1}<\cdots<\alpha_{i-1}<\alpha, \alpha<\alpha_{i+1}<\cdots<\alpha_{m}<M\right) .
\end{aligned}
$$

Hence we have that $\left(F_{*}^{0}\left(\beta_{1}\right), \delta^{0}\right)$ is acyclic for all $\beta_{1}$ and so

$$
H_{*}\left(F_{*}^{0}, \delta^{0}\right)=H_{*}\left(E_{*}^{0}(\alpha ; 0), d^{0}\right) .
$$

Now note that $E_{*}^{0}(\alpha ; 0)$ is isomorphic to the tensor product

$$
\left(E_{*}^{0}(\alpha ; 0), d^{0}\right) \cong\left(C_{*}([0, \alpha]), \partial\right) \otimes\left(\mathrm{ND}_{*}(\alpha), \partial\right),
$$

an isomorphism which follows directly from (4.2.1). Thus

$$
F^{1}=H_{*}\left(F_{*}^{0}, \delta^{0}\right) \cong H_{*}([0, \alpha]) \otimes\left(H_{*}(K)\right)^{\otimes r} .
$$

Since all the homology on the right is concentrated in the 0th-filtered piece of $F^{1}$, the spectral sequence $\left(F^{s}, \delta^{S}\right)$ has collapsed at $F^{1}$. So

$$
E^{1} \cong \bigoplus_{\alpha} H_{*}([0, \alpha]) \otimes\left(H_{*}(K)\right)^{\otimes c(\alpha)}
$$

where $c(\alpha)$ denotes the number of connected components of $\alpha$.

If $V=\bigoplus_{n} V_{n}$ is a graded vector space then the total dimension of $V$, written $\operatorname{dim}(V)$, is defined to be the sum of the dimensions of the $V_{n}$.

Corollary 4.4.6. Let $\kappa$ be the total dimension of $H(K)$. Then the total dimension of $E^{1}$ is given by

$$
\operatorname{dim}\left(E^{1}\right)=\prod_{i=0}^{n-1}(\kappa+i|G|)
$$


Proof. By Theorem 4.4.5 we have

$$
\operatorname{dim}\left(E^{1}\right)=\sum_{r_{0}} \sum_{\substack{\alpha \\ r(\alpha)=r_{0}}} \operatorname{dim}(H([0, \alpha])) \kappa^{r_{0}} .
$$

Let $\hat{\alpha}$ be a partition in $\Pi_{n}$ with $r_{0}$ blocks of sizes $b_{1}, \ldots, b_{r_{0}}$. Then the number of elementary $K$-digraphs whose connected components are the blocks of $\hat{\alpha}$ is $\prod_{j}|G|^{b_{j}-1}$. Also if $\alpha$ is such a $K$-digraph then $\operatorname{dim}(H([0, \alpha]))=$ $\prod_{j}\left(b_{j}-1\right)$ !. Note that $\operatorname{dim}(H([0, \alpha]))$ is the number of permutations in $S_{n}$ whose partitions into disjoint cycles is $\hat{\alpha}$. Thus

$$
\begin{aligned}
\operatorname{dim}\left(E^{1}\right) & =n ! Z\left(S_{n}\right)\left[x_{t} \rightarrow|G|^{t-1} \kappa\right] \\
& =n !|G|^{n} Z\left(S_{n}\right)\left[x_{t} \rightarrow \frac{\kappa}{|G|}\right] .
\end{aligned}
$$

So

$$
\operatorname{dim}\left(E^{1}\right)=n !|G|^{n}\left(\begin{array}{c}
\frac{\kappa}{|G|}+n-1 \\
n
\end{array}\right) .
$$

If we substitute $t=1$ in Theorem 4.1 .4 we have

$$
\begin{aligned}
\operatorname{dim}\left(H_{*}\left(D_{n}(G, K)\right)\right) & =\prod_{i=0}^{n-1}(\kappa+i|G|) \\
& =n !|G|^{n}\left(\begin{array}{c}
\kappa \\
|G| \\
n
\end{array}\right)=\operatorname{dim}\left(E^{1}\right) .
\end{aligned}
$$

Since $H_{*}\left(D_{n}(G, K)\right)=E^{\infty}$ we see from (4.4.8) that our spectral sequence has collapsed at $E^{1}$ which proves the following theorem.

Theorem 4.4.9. As a graded vector space, $H_{*}\left(D_{n}(G, K)\right)$ is isomorphic to

$$
H_{*}\left(D_{n}(G, K)\right) \cong \bigoplus_{\alpha} H_{*}([0, \alpha]) \otimes\left(H_{*}(K)\right)^{\otimes c(\alpha)},
$$

where the sum on the right is over all elementary digraphs $\alpha$.

4.5. The homology of $L_{n}(V)$. Recall the set-up of $\S 3$ in which $\varphi: G \rightarrow \operatorname{GL}(V)$ is a finite-dimensional faithful complex representation of $G$ which does not contain the trivial representation. Notation and terminology will be as in that section.

Let $\mathscr{U}_{1}, \ldots, \mathscr{U}_{s}$ be the coatoms in $\mathscr{V}$, i.e., the maximal subspaces in $\mathscr{V} \backslash \mathbb{C}^{d}$. Let $\mathscr{K}(\varphi)$ denote the complement of $\left(\bigcup_{i=1}^{s} \mathscr{U}_{i}\right)$ in $\mathbb{C}^{d}$. In this section we will compute the homology of $\Lambda_{n}(\varphi)$ in terms of the homology of $\mathscr{K}(\varphi)$. Our main tool will be the theorem of Goresky and Macpherson [8] which appears below.

Let $A_{1}, \ldots, A_{t} \subseteq \mathbb{R}^{m}$ be a finite set of affine subspaces (of possibly various dimensions) in Euclidean space and let

$$
M=\mathbb{R}^{m} \backslash\left(\bigcup_{i=1}^{t} A_{i}\right) .
$$


Associated to this collection $A_{1}, \ldots, A_{t}$ there is a poset $\mathscr{P}$ whose elements $v$ correspond to the flats

$$
|v|=A_{i_{1}} \cap \cdots \cap A_{i_{r}}
$$

(ordered by inclusion) with a maximal element $\hat{1}$ appended which corresponds to the space $\mathbb{R}^{m}$. There is a natural ranking function $d$ on $\mathscr{P}$ given by

$$
d(v)=\operatorname{dim}_{\mathbb{R}}(|v|) .
$$

Theorem 4.5.1 (Goresky and Macpherson [8, Theorem 1.3A, p. 238]). The homology of $M$ is given by

$$
H_{i}(M ; \mathbb{Z})=\bigoplus_{v \in \mathscr{P}} H_{m-d(v)-i-1}([0, v]) .
$$

Note in this theorem that the homology on the left is homology of the affine variety $M$ whereas homology on the right is homology of the poset $[0, v]$. Also note that the exact form of the subscript on the right, $m-d(v)-i-1$, is slightly different than what appears in [8]. This is because we use a different convention for determining the rank on poset homology.

We will be using Theorem 4.5.1 in the case that $\mathbb{R}^{m}$ is a complex space $\mathbb{C}^{m / 2}$ and each $A_{i}$ is a complex subspace. One can apply the theorem as stated above although it is important to remember that $d(v)$ is the real dimension of $|v|$.

Let $P(\mathscr{K}(\varphi) ; t)$ and $P\left(\Lambda_{n}(\varphi) ; t\right)$ denote the Poincare series for the homology groups of $\mathscr{K}(\varphi)$ and $\Lambda_{n}(\varphi)$ respectively, i.e.,

$$
\begin{aligned}
& P(\mathscr{K}(\varphi) ; t)=\sum_{i} \operatorname{dim}\left(H_{i}(\mathscr{K}(\varphi) ; \mathbb{Z})\right) t^{i} \\
& P\left(\Lambda_{n}(\varphi) ; t\right)=\sum_{i} \operatorname{dim}\left(H_{i}\left(\Lambda_{n}(\varphi) ; \mathbb{Z}\right)\right) t^{i}
\end{aligned}
$$

Theorem 4.5.3. For any $n$ and $\varphi$ we have

$$
t P\left(\Lambda_{n}(\varphi) ; t\right)=\prod_{i=0}^{n-1}\left(t P(\mathscr{K}(\varphi) ; t)+i|G| t^{2 d-1}\right) .
$$

Proof. We will apply Theorem 4.5.1 to compute $P\left(\Lambda_{n}(\varphi) ; t\right)$. Let $v \in D_{n}(G)$. Then $v$ determines a pair $\eta(v)=\left(C_{0}, \gamma\right)$, where $C_{0}$ is the distinguished component of $v$ and $\gamma$ is the partition of $\mathbf{n} \backslash C_{0}$ whose blocks are the nondistinguished connected components of $v$. In each block $C_{i}$ of $\gamma$ pick out the smallest element $u_{i}$ and let $c_{i}$ denote the size of $C_{i}$. Our first step is to compute $\sum\left(C_{0}, \gamma\right)$ which is the contribution to the right-hand side of (4.5.2) made by all $v$ which give us the pair $\left(C_{0}, \gamma\right)$.

Suppose that $\eta(v)=\left(C_{0}, \gamma\right)$ and that the point $u_{i}$ in $C_{i}$ is labelled by the subgroup $H_{i}$ which corresponds to the subspace $V_{i}=V_{H_{i}}$. Then

$$
\operatorname{dim}_{\mathbb{R}}(|v|)=\sum_{l=1}^{s} \operatorname{dim}_{\mathbb{R}}\left(V_{l}\right)
$$


( $s$ equals the number of blocks of $\gamma$ ). So,

$$
\begin{aligned}
\sum\left(C_{0}, \gamma\right) & =t \sum_{\substack{v \\
\eta(v)=\left(C_{0}, \gamma\right)}} \sum_{i} \operatorname{dim}\left(H_{2 n d-2\left(\sum \operatorname{dim}_{\mathbf{R}}\left(V_{l}\right)\right)-i-1}([\hat{0}, v]) t^{i}\right. \\
& =\sum_{\substack{v \\
\eta(v)=\left(C_{0}, \gamma\right)}} \sum_{j} \operatorname{dim}\left(H_{j}([\hat{0}, v])\right) t^{2 n d-2\left(\sum \operatorname{dim}_{\mathbf{R}}\left(V_{l}\right)\right)-j}
\end{aligned}
$$

Note that the interval $[\hat{0}, v]$ in $D_{n}(G)$ is isomorphic to

$$
[\hat{0}, v] \cong D_{c_{0}}(G, K) \times\left\{\prod_{l=1}^{s} \Omega_{c_{l}}\left(H_{l}, K_{l}\right)\right\},
$$

where $K_{l}$ is the interval $\left[E, H_{l}\right]$ in $K$. Thus

$$
\sum\left(C_{0}, \gamma\right)=\sum_{v}\left\{t^{2 c_{0} d} P\left(D_{c_{0}}(G, K) ; t^{-1}\right)\right\}\left\{\prod_{l=1}^{s} X_{l}\right\},
$$

where $X_{l}=t^{2 c_{l} d-2 \operatorname{dim}_{\mathbf{R}}\left(V_{l}\right)} P\left(\Omega_{c_{l}}\left(H_{l}, K_{l}\right) ; t^{-1}\right)$. From Theorem 4.1.3 we have

$$
X_{l}=\left\{\left(c_{l}-1\right) !\left(|G| t^{(2 d-1)}\right)^{\left(c_{l}-1\right)}\right\} t^{2 d-\operatorname{dim}_{\mathbf{R}}\left(V_{l}\right)} P\left(\left[0, H_{l}\right] ; t^{-1}\right) .
$$

Here $P\left(\left[0, H_{l}\right] ; t^{-1}\right)$ is the Poincare polynomial (in the variable $t^{-1}$ ) for the homology of the interval $\left[0, H_{l}\right]$ in $K$.

Next observe that if $\eta(v)=\left(C_{0}, \gamma\right)$ then $v$ is completely determined by the choice of subgroups $H_{1}, \ldots, H_{s}$ labelling $u_{1}, \ldots, u_{s}$. These choices can be made arbitrarily subject to the constraint $H_{l} \neq G$. Also, by Theorem 4.1.4 we have

$$
t^{2 c_{0} d} P\left(D_{c_{0}}(G, K) ; t^{-1}\right)=\prod_{i=0}^{c_{0}-1}\left(t^{2 d} P\left(K ; t^{-1}\right)+i|G| t^{2 d-1}\right) .
$$

Thus

$$
\sum\left(C_{0}, \gamma\right)=\left\{\prod_{i=0}^{c_{0}-1}\left(t^{2 d} P\left(K ; t^{-1}\right)+i|G| t^{2 d-1}\right)\right\}\left\{\prod_{l=1}^{s} Y_{l}\right\}
$$

where

$$
\begin{aligned}
Y_{l}= & \left(c_{l}-1\right) !\left(t^{(2 d-1)}|G|\right)^{c_{l}-1} \\
& \cdot t\left\{\sum_{\mathscr{U}_{l} \neq G} \sum_{i} \operatorname{dim}\left(H_{i}\left(\left[E, \mathscr{U}_{l}\right]\right)\right) t^{2 d-\operatorname{dim}_{\mathbf{R}}\left(V_{\mathscr{U}_{l}}\right)-i-1}\right\} .
\end{aligned}
$$

Note that if $\mathscr{U}=G$ then

$$
\sum_{i} \operatorname{dim}\left(H_{i}([E, \mathscr{U}])\right) t^{2 d-\operatorname{dim}_{\mathbf{R}}\left(V_{\mathcal{U}}\right)-i-1}=t^{2 d-1} P\left(K ; t^{-1}\right) .
$$


Thus

$$
\begin{gathered}
t \sum_{\mathscr{U}_{l} \neq G} \sum_{i} \operatorname{dim}\left(H_{i}\left(\left[E, \mathscr{U}_{l}\right]\right)\right) t^{2 d-\operatorname{dim}_{\mathbf{R}}\left(V_{\mathscr{U}_{l}}\right)-i-1} \\
=t P(\mathscr{K}(\varphi) ; t)-t^{2 d} P\left(K ; t^{-1}\right)
\end{gathered}
$$

So, from (4.5.4) we have

$$
\begin{aligned}
\sum\left(C_{0}, \gamma\right)= & \left\{\prod_{i=0}^{c_{0}-1}\left(t^{2 d} P\left(K ; t^{-1}\right)+i|G| t^{2 d-1}\right)\right\} \\
& \cdot \prod_{l=1}^{s}\left(\left(c_{l}-1\right) !\left(t^{2 d-1}|G|\right)^{c_{l}-1}\left(t P(\mathscr{K}(\varphi) ; t)-t^{2 d} P\left(K ; t^{-1}\right)\right)\right) .
\end{aligned}
$$

Note that $\sum\left(C_{0}, \gamma\right)$ depends on $P\left(K ; t^{-1}\right)$ as well as $P(\mathscr{K}(\varphi) ; t)$. However we shall see that when we sum the $\sum\left(C_{0}, \gamma\right)$ the dependence on $P\left(\mathscr{K} ; t^{-1}\right)$ disappears leaving the expression on the right-hand side of Theorem 4.5.3 which depends only on $P(\mathscr{K}(\varphi) ; t)$.

Fix $c_{0}, c_{1}, \ldots, c_{s}$ and sum $\sum\left(C_{0}, \gamma\right)$ over all pairs $\left(C_{0}, \gamma\right)$, where $\left|C_{0}\right|=$ $c_{0}$ and where $\gamma$ has blocks of size $c_{1}, \ldots, c_{s}$. Call this sum $\sigma\left(c_{0} ; c_{1}, \ldots, c_{s}\right)$. If $j_{i}$ is the number of $c_{l}(l \geq 1)$ which equal $i$ then we have

$$
\begin{aligned}
\sigma\left(c_{0} ;\right. & \left.c_{1}, \ldots, c_{s}\right) \\
= & \left(\begin{array}{l}
n \\
c_{0}
\end{array}\right)\left\{\prod_{i=0}^{c_{0}-1}\left(t^{2 d} P\left(k ; t^{-1}\right)+i|G| t^{2 d-1}\right)\right\} \cdot\left(\frac{\left(n-c_{0}\right) !}{\prod_{i}(i !)^{j_{i}} j_{i} !}\right) \\
& \cdot\left\{\prod_{l=1}^{s}\left(c_{l}-1\right) !\left(t^{2 d-1}|G|\right)^{c_{l}}\left(\frac{t P(\mathscr{K}(\varphi) ; t)-t^{2 d} P\left(K ; t^{-1}\right)}{t^{2 d-1}|G|}\right)\right\} .
\end{aligned}
$$

Let $u, v, w$ denote

$$
u=t^{2 d} P\left(K ; t^{-1}\right), \quad v=t P(\mathscr{K}(\varphi) ; t), \quad w=t^{2 d-1}|G| .
$$

Note that

$$
\prod_{i=0}^{c_{0}-1}\left(t^{2 d} P\left(K ; t^{-1}\right)+i|G| t^{2 d-1}\right)=w^{c_{0}} c_{0} !\left(\begin{array}{c}
\frac{u}{w}+c_{0}-1 \\
c_{0}
\end{array}\right) .
$$

Also

$$
\begin{aligned}
\left(\frac{\left(n-c_{0}\right) !}{\prod_{i}(i !)^{j_{i}} j_{i} !}\right) & \left\{\prod_{l=1}^{s}\left(c_{l}-1\right) !\left(t^{2 d-1}|G|\right)^{c_{l}}\left(\frac{t P(\mathscr{K}(\varphi) ; t)-t^{2 d} P\left(K ; t^{-1}\right)}{t^{2 d-1}|G|}\right)\right\} \\
& =w^{n-c_{0}} \sum_{\tau} Z(\tau)\left[x_{t} \leftarrow\left(\frac{v}{w}-\frac{u}{w}\right)\right],
\end{aligned}
$$


where the sum on $\tau$ is over all permutations in $S_{n-c_{0}}$ which have cycles of lengths $c_{1}, \ldots, c_{s}$. Substituting (4.5.6) and (4.5.7) into (4.5.5) we have

$$
\begin{aligned}
t P\left(\Lambda_{n}(\varphi) ; t\right) & =\sum_{\left(c_{0}, \ldots, c_{s}\right)} \sigma\left(c_{0} ; c_{1}, \ldots, c_{s}\right) \\
& =\sum_{c_{0}} n ! w^{n}\left(\begin{array}{c}
\frac{u}{w}+c_{0}-1 \\
c_{0}
\end{array}\right)\left(\begin{array}{c}
\frac{v}{w}-\frac{u}{w}+\left(n-c_{0}\right)-1 \\
\left(n-c_{0}\right)
\end{array}\right) .
\end{aligned}
$$

Noting that $\left(\begin{array}{c}\lambda+a-1 \\ a\end{array}\right)=\left(\begin{array}{c}-\lambda \\ a\end{array}\right)(-1)^{a}$ we have

$$
\begin{aligned}
t P\left(\Lambda_{n}(\varphi) ; t\right) & =n ! w^{n}\left(\begin{array}{c}
\frac{-u}{w}+\left(\frac{u}{w}-\frac{v}{w}\right) \\
n
\end{array}\right)(-1)^{n} \\
& =n ! w^{n}\left(\begin{array}{c}
\frac{v}{w} \\
n
\end{array}\right)(-1)^{n}=n ! w^{n}\left(\begin{array}{c}
\frac{v}{w}+n-1 \\
n
\end{array}\right) \\
& =\prod_{i=0}^{n-1}(v+i w)=\prod_{i=0}^{n-1}\left(t P(\mathscr{K}(\varphi) ; t)+i|G| t^{2 d-1}\right),
\end{aligned}
$$

which completes the proof.

Note the striking similarity between Theorems 4.1 .4 and 4.5.3. It seems plausible that there might be some simple way to derive Theorem 4.5 .3 from Theorem 4.1.4. However the author knows of no proof that is more direct than the one given here.

5. The ACtion of $G$ wr $S_{n}$ ON THE hoMOlogy of $D_{n}(G, K)$

5.1. The wreath product $G$ wr $S_{n}$. Let $G$ wr $S_{n}$ denote the wreath product of $G$ over $S_{n}$ (see James and Kerber [11] for more background on wreath products). Throughout this section we will assume that $n, G, K$ are fixed and we will let $\mathscr{G}$ denote $G$ wr $S_{n}$. We will think of $\mathscr{G}$ as the set of $n \times n$ matrices with entries from $G \cup\{0\}$ which have exactly one nonzero entry per row and column. If $\sigma \in \mathscr{G}$.we let $\hat{\sigma}$ denote the underlying permutation in $S_{n}$, i.e., $\hat{\sigma}$ is obtained from $\sigma$ by changing every nonzero entry to 1 . Also we let $g(\sigma ; i)$ denote the element of $G$ that occupies the unique nonzero entry in row $i$ of $\sigma$.

There is an action of $\mathscr{G}$ on the set $\Omega_{n}(G, K)$. Let $\delta$ be an element of $\Omega_{n}(G, K)$ with edge labels $H_{i j}$ and let $\sigma$ be an element of $\mathscr{G}$. Define $\sigma \cdot \delta$ to the digraph with edge labels $H_{u, v}^{\prime}$ given by

$$
H_{i \hat{\sigma}, j \hat{\sigma}}^{\prime}=g(\sigma ; j) H_{i, j} g(\sigma ; i)^{-1} \text {. }
$$

It is straightforward to check that $\sigma \cdot \delta$ is a $K$-digraph and that each $\sigma \in \mathscr{G}$ is an automorphism of the poset $\Omega_{n}(G, K)$ which maps $D_{n}(G, K)$ to $D_{n}(G, K)$. So we have a representation of $\mathscr{G}$ as a group of automorphisms of $D_{n}(G, K)$. This in turn gives an action of $\mathscr{G}$ on the homology of $D_{n}(G, K)$. We denote the character of the representation of $\mathscr{G}$ on $H_{i}\left(D_{n}(G, K)\right)$ by $\beta_{n, i}$. Our goal in this section is to derive information about the characters $\beta_{n, i}$. Our main 
result here will express $\beta_{n, i}$ as a sum of induced characters. For the rest of $\S 5.1$ we will catalogue some further information about the group $\mathscr{G}$.

Let $\mathscr{C}_{1}, \ldots, \mathscr{C}_{\kappa}$ be the conjugacy classes of $G$. From each conjugacy class $\mathscr{C}$ choose an element $g(\mathscr{C})$ which will remain fixed for the rest of this section. Let $\sigma \in \mathscr{G}$ and let $Y=\left(y_{1}, \ldots, y_{s}\right)$ be a cycle of $\sigma$ (i.e., a cycle of $\hat{\sigma}$ ). We say $Y$ has conjugacy type $\mathscr{C}$ if

$$
\left\{\prod_{i=1}^{s} g\left(\sigma ; y_{i}\right)\right\} \in \mathscr{C} .
$$

Note that the actual group element defined by the product in (5.1.1) depends on the cyclic order of $\left(y_{1}, \ldots, y_{s}\right)$. But this dependence is only up to conjugacy in $G$, hence the conjugacy type of $Y$ is well defined. For each $u \in\{1,2, \ldots, n\}$ and each $s \in\{1,2, \ldots, \kappa\}$ let $m_{\sigma}(u, s)$ denote the number of $u$-cycles of $\sigma$ with conjugacy type $\mathscr{C}_{s}$. The type of $\sigma, \tau(\sigma)$, is the $m s$-tuple

$$
\tau(\sigma)=\left(m_{\sigma}(u, s)\right)
$$

It is well known (see, for example, James and Kerber [11]) that two elements of $\mathscr{G}$ are conjugate if and only if they have the same type.

An element of $\mathscr{G}$ is standard if each cycle has at most one nonidentity element of $G$. It is clear that every element of $\mathscr{G}$ is conjugate to a standard element. Let $\sigma$ be standard and let $Y=\left(y_{1}, y_{2}, \ldots, y_{u}\right)$ be a cycle of $\sigma$. If $Y$ contains a nonidentity element $g \in G$ then reorder $Y$ cyclically so that $g$ occurs in the $y_{u}$ th row and $y_{1}$ st column of $\sigma$. Define $\beta(Y)=y_{1}$ (the beginning of $Y$ ) and $\varepsilon(Y)=y_{u}$ (the end of $Y$ ). The submatrix of $\sigma$ corresponding to $Y$ looks like

$$
\begin{array}{r}
\beta(Y)=y_{1} \\
y_{2} \\
\vdots \\
\varepsilon(Y)=y_{u}
\end{array}\left(\begin{array}{ccccc}
0 & e & 0 & \cdots & 0 \\
0 & 0 & e & \cdots & 0 \\
& & \vdots & & \\
g & 0 & 0 & \cdots & 0
\end{array}\right)
$$

5.2. The action of $\mathscr{G}$ on $\mathscr{I}_{n}(G)$. Recall from $\S 4.3$ that $\mathscr{I}_{n}(G)$ is the poset of elementary digraphs in $D_{n}(G, K)$, i.e., those digraphs in which every (nonempty) edge label is a single group element. In this subsection we will derive some facts about the action of $\mathscr{G}$ on $\mathscr{F}_{n}(G)$ and on the homology groups of the intervals in $\mathscr{I}_{n}(G)$.

Let $\alpha$ be an element of $\mathscr{I}_{n}(G)$ which is fixed by some $\sigma \in \mathscr{G}$. Then $\sigma$ permutes the connected components of $\alpha$. Write $\alpha$ as the disjoint union

$$
\alpha=\alpha_{1} \cup \alpha_{2} \cup \cdots \cup \alpha_{s},
$$

where each $\alpha_{i}$ is an orbit of connected components of $\alpha$. Each $\alpha_{i}$ is a union of some number $t_{i}$ of connected components,

$$
\alpha_{i}=C_{i}^{(1)} \cup C_{i}^{(2)} \cup \cdots \cup C_{i}^{\left(t_{i}\right)},
$$

where $\sigma$ maps $C_{i}^{(u)}$ to $C_{i}^{(u+1)}$ (here $u+1$ is taken $\bmod t_{i}$ ). Let $\sigma_{i}$ denote the restriction of $\sigma$ to $\alpha_{i}$. 
We can decompose $\sigma_{i}$ into disjoint cycles

$$
\sigma_{i}=Y_{i}^{(1)} Y_{i}^{(2)} \cdots Y_{i}^{\left(j_{i}\right)} \text {. }
$$

The length of each cycle $Y_{i}^{(l)}$ is a multiple $d_{i}^{(l)} t_{i}$ of $t_{i}$. Also let $\mathscr{D}_{i}^{(l)}$ denote the conjugacy type of $Y_{i}^{(l)}$. We call the sets $\left\{t_{i}\right\},\left\{j_{i}\right\},\left\{d_{i}^{(l)}\right\}$, and $\left\{\mathscr{D}_{i}^{(l)}\right\}$ the $\alpha$-invariants of $\sigma$ (defined for any pair $(\alpha, \sigma)$ where $\sigma \cdot \alpha=\alpha$ ).

Defintion 5.2.1. Let notation be as above. We say $\sigma_{i}$ is $\alpha$-uniform if $d_{i}^{(1)}=$ $d_{i}^{(2)}=\cdots=d_{i}^{\left(j_{i}\right)}$. We say $\sigma$ is $\alpha$-uniform if each $\sigma_{i}$ is $\alpha$-uniform.

Lemma 5.2.2. Let notation be as above and suppose $\sigma_{i}$ is $\alpha$-uniform. Then $\mathscr{D}_{i}^{(1)}=\mathscr{D}_{i}^{(2)}=\cdots=\mathscr{D}_{i}^{\left(j_{i}\right)}$.

Proof. Assume $\sigma_{i}$ is $\alpha$-uniform. Let $a_{i}^{(u)}$ and $a_{i}^{(v)}$ be arbitrarily chosen elements of $Y_{i}^{(u)} \cap C_{i}^{(1)}$ and $Y_{i}^{(v)} \cap C_{i}^{(1)}$. Let $g$ and $h$ be the products of the entries of $Y_{i}^{(u)}$ and $Y_{i}^{(v)}$ starting with $a_{i}^{(u)}$ and $a_{i}^{(v)}$ respectively. Thus $g \in \mathscr{D}_{i}^{(u)}$ and $h \in \mathscr{D}_{i}^{(v)}$. Last, let $x$ be the label on the edge from $a_{i}^{(u)}$ to $a_{i}^{(v)}$.

If we apply $\sigma_{i}^{d_{i} t_{i}}$ to the edge from $a_{i}^{(u)}$ to $a_{i}^{(v)}$ we obtain the same edge but with label changed from $x$ to $h x g^{-1}$. Since $\sigma \cdot \alpha=\alpha$ we have

$$
h x g^{-1}=x \text { so } h=x g x^{-1} \text {. }
$$

Thus $\mathscr{D}_{i}^{(u)}=\mathscr{D}_{i}^{(v)}$ which completes the proof.

Lemma 5.2.3. Assume that $\sigma \cdot \alpha=\alpha$ and that $\left\{j_{i}\right\},\left\{d_{i}^{(u)}\right\}$, and $\left\{\mathscr{D}_{i}^{(u)}\right\}$ are the parameters of $(\sigma, \alpha)$. Let $T_{r}$ denote the trace of $\sigma$ acting on the $r$ th homology group of the interval $[0, \alpha]$. Then

$$
T_{r}= \begin{cases}(-1)^{n-c(\alpha)} \prod_{i}\left(-d_{i}\right)^{j_{i}-1}\left(j_{i}-1\right) ! \mu(d) & \text { if } \sigma \text { is } \alpha \text {-uniform and } \\ 0 \text { otherwise. } & r=n-c(\alpha)-1,\end{cases}
$$

Proof. Observe that the interval $[0, \alpha]$ in $\mathcal{F}_{n}(G)$ is isomorphic to the interval $[0, \Pi(\alpha)]$ in $\Pi_{n}$. So $[0, \alpha]$ is C-M of rank $n-c(\alpha)$ which shows that $T_{r}=0$ unless $r=n-c(\alpha)-1$. It also implies that

$$
T_{n-c(\alpha)}=(-1)^{n-c(\alpha)} \mu_{[0, \alpha]_{\sigma}}(0, \alpha),
$$

where $[0, \alpha]_{\sigma}$ denotes the subposet of $[0, \alpha]$ consisting of all elements fixed by $\sigma$.

It is easy to see that

$$
[0, \alpha]_{\sigma} \cong\left[0, \alpha_{1}\right]_{\sigma_{1}} \times \cdots \times\left[0, \alpha_{s}\right]_{\sigma_{s}}
$$

and that $\left[0, \alpha_{i}\right]_{\sigma_{i}}$ is isomorphic to the lattice of partitions of $C_{i}^{(1)}$ fixed by $\sigma_{i}^{t_{i}}$ (note that $\sigma_{i}^{t_{i}}$ acts on $C_{i}^{(1)}$ ). The rest of Lemma 5.2.3 now follows immediately from $[9$, Theorem $]$. 
We will need a bit more information about the elementary $K$-digraphs fixed by $\sigma$ in the case the $\sigma$ is standard. Assume henceforth that $\sigma$ is standard, that $\sigma \cdot \alpha=\alpha$, and that $\sigma$ is $\alpha$-uniform. Let $\left\{t_{i}\right\},\left\{j_{i}\right\},\left\{d_{i}\right\}$, and $\left\{\mathscr{D}_{i}\right\}$ be the $\alpha$-parameters of $\sigma$. We will examine the structure of a particular $\alpha_{i}$.

As above, write $\sigma_{i}=Y_{i}^{(1)} Y_{i}^{(2)} \ldots Y_{i}^{\left(j_{i}\right)}$ and let $C_{i}^{(1)}, C_{i}^{(2)}, \ldots, C_{i}^{\left(t_{i}\right)}$ be the connected components of $\alpha_{i}$ written so that $\sigma_{i}\left(C_{i}^{(u)}\right)=C_{i}^{(u+1)}$ and so that $\beta\left(Y_{i}^{(1)}\right) \in C_{i}^{(1)}$. Define $z(1), z(2), \ldots, z\left(d_{i}\right) \in C_{i}^{(1)}$ by

$$
z(j)=\sigma^{(j-1) t_{i}} \beta\left(Y_{i}^{(1)}\right)
$$

and let $y(u)$ be an arbitrarily chosen element of $Y_{i}^{(u)} \cap C_{i}^{(1)}$. A diagram depicting $\alpha_{i}$ appears below:

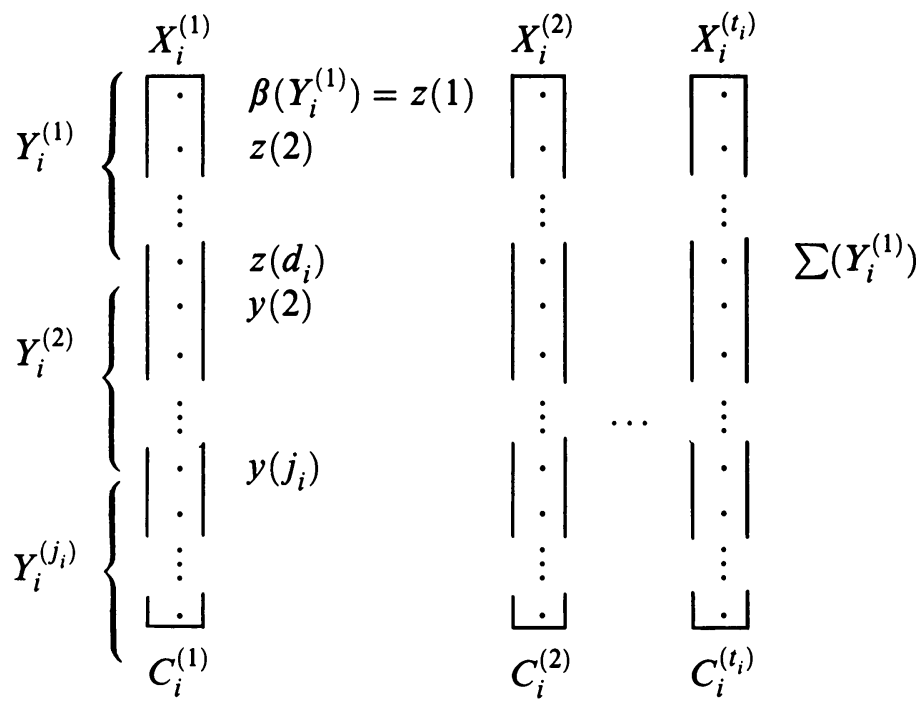

FIGURE 5.2.4

Lemma 5.2.5. Let $h$ be the label on the edge from $z(1)$ to $z(2)$ and let $u^{(j)}$ be the label on the edge from $z(1)$ to $y(j)$. Let $g=g\left(\mathscr{D}_{i}\right)$. Then

(1) $h^{d_{i}}=g^{-1}$,

(2) $u^{(j)} \in C_{G}(g)$.

Conversely, a choice of $h$ such that $h^{d_{i}}=g^{-1}$ to label the edge from $z(1)$ to $z(2)$ and choices of $u^{(j)} \in C_{G}(g)$ to label the edges from $z(1)$ to $y(j)$ determines an elementary $k$-digraph $\alpha_{i}$ which is fixed by $\sigma_{i}$.

Proof. First we prove that $h$ is a $d_{i}$ th root of $g^{-1}$. Observe that the edge from $z(s)$ to $z(s+1)$ is labelled $h$ in $\alpha$ (by invariance of $\alpha$ under $\sigma$ and by the standardness of $\sigma)$. Thus the edge from $z\left(d_{i}\right)$ to $z(1)$ is labelled $h^{-\left(d_{i}-1\right)}$.

If we apply $\sigma^{t_{i}}$ to the edge from $z\left(d_{i}-1\right)$ to $z\left(d_{i}\right)$ we obtain the edge from $z\left(d_{i}\right)$ to $z(1)$ labelled by $h g$. By invariance of $\alpha$ under $\sigma$ we have

$$
h g=h^{-\left(d_{i}-1\right)}
$$

as desired. 
Next we show that $u^{(j)}$ centralizes $g$. Note that if $\sigma^{t_{i} d_{i}}$ is applied to the edge from $y(1)$ to $y(j)$ the result is the edge from $y(1)$ to $y(j)$ but with label changed to $g^{-1} u(j) g$. The result follows.

The converse statements are straightforward and are left to the reader.

5.3. The action of $\mathscr{G}$ on $H_{*}\left(\Omega_{n}(G, K)\right)$. In this section we compute the action of $\mathscr{G}=G$ wr $S_{n}$ on the homology of $\Omega_{n}(G, K)$. Our main result is an interesting generalization of a result due to Stanley. Consider the case where $G$ is the trivial group. It is straightforward to see that $\Omega_{n}(G, K)$ is isomorphic to the partition lattice in this case. Also in this case $\mathscr{G}^{n}$ is just $S_{n}$ and so the action of $\mathscr{G}$ on $H_{*}\left(\Omega_{n}(G, K)\right)$ is the action of $S_{n}$ on $H_{*}\left(\Pi_{n}\right)$. Stanley proved the following elegant result.

Let $C_{n}$ be the cyclic group in $S_{n}$ generated by $\tau_{n}=(1,2, \ldots, n)$ and let $z_{n}$ be the linear character of $C_{n}$ defined by

$$
z_{n}\left(\tau_{n}\right)=e^{2 \pi i / n} \text {. }
$$

Theorem 5.3.1 (Stanley [16]). Let $\chi^{(n)}$ be the character of $S_{n}$ acting on the unique nonvanishing reduced homology group of $\Pi_{n}$. Then

$$
\chi^{(n)}=\operatorname{sgn} \cdot \operatorname{ind}_{C_{n}}^{S_{n}}\left(z_{n}\right) \text {. }
$$

To understand how this result generalizes in our situation first note that $C_{n} \times G$ can be identified as a subgroup of $\mathscr{G}$. The matrix in this subgroup corresponding to $\left(\tau_{n}^{j}, g\right)$ is the one which has entry $g$ wherever the permutation matrix corresponding to $\tau_{n}^{j}$ has a 1 .

$G$ acts on the graded module $H_{*+(n-1)}(K)$. Here $H_{*+(n-1)}(K)$ is identical as a module to $H_{*}(K)$ except that the grading has been shifted up by $n-1$. So $C_{n} \times G$ acts on $\mathbb{C} \otimes H_{*+(n-1)}(K)=H_{*+(n-1)}(K)$ by the tensor product action:

$$
\left(\tau_{n}^{j}, g\right)(v \otimes u)=\left(z_{n}\left(\tau_{n}^{j}\right) v\right) \otimes(g \cdot u) .
$$

Our main result in this section is the following generalization of Theorem 5.3.1.

Theorem 5.3.2. Let $\chi_{*}^{(n)}(G, K)$ be the graded representation of $\mathscr{G}$ on the homology of $\Omega_{n}(G, K)$. Then

$$
\chi_{*}^{(n)}(G, K)=\operatorname{sgn} \cdot \operatorname{ind}_{C_{n} \times G}^{G \text { wr } S_{n}}\left(z_{n} \otimes H_{*+(n-1)}(K)\right) .
$$

Proof. We will prove this result by computing the character of $\mathscr{G}$ on $H_{*}\left(\Omega_{n}(G, K)\right)$. Recall that in $\S 4.1$ we had established an isomorphism of graded vector spaces between $H_{*}\left(\Omega_{n}(G, K)\right)$ and $M=\bigoplus_{\alpha \in \mathcal{F}_{n}^{(1)}(G)} H_{*}([0, \alpha]) \otimes$ $H_{*}(K)$. Here $\mathscr{F}_{n}^{(1)}(G)$ was the set of connected $G$-digraphs. Recall that the isomorphism between $H_{*}\left(\Omega_{n}(G, K)\right)$ and $M$ came from the fact that $\mathscr{I}_{n}^{(1)}(G)$ is the set of complements to the digraph $z$ whose only edges are loops at each point labelled $G$. Note that $z$ is invariant under $\mathscr{G}$. Because of this it is easy to check that the isomorphism between $H_{*}\left(\Omega_{n}(G, K)\right)$ and $M$ is $\mathscr{G}$-equivariant. 
So the character of $\mathscr{G}$ on $H_{*}\left(\Omega_{n}(G, K)\right)$ agrees with the character on $M$. We will compute the latter character.

Let $\sigma$ be an element of $\mathscr{G}$. We want to compute the trace of $\sigma$ acting on $M$. This trace is a class function so we may choose $\sigma$ up to conjugation in $\mathscr{G}$. In particular, we will assume that $\sigma$ is standard and has a disjoint cycle decomposition $\sigma=Y^{(1)} \cdots Y^{(j)}$, where $1=\beta\left(Y^{(1)}\right)$.

It is important to understand how $\mathscr{G}$ acts on $M$. This action is induced from an action of $\mathscr{G}$ on the associated chain complex

$$
M^{\prime}=\bigoplus_{\alpha \in \mathcal{F}_{n}^{(1)}(G)} C_{*}([0, \alpha]) \otimes C_{*}(K)
$$

To understand how $\mathscr{G}$ acts on $M^{\prime}$ one must embed $M^{\prime}$ in $C_{*}\left(D_{n}(G, K)\right)$ using the map $\psi: M^{\prime} \rightarrow C_{*}\left(D_{n}(G, K)\right)$ defined by

$$
\begin{gathered}
\psi\left(\left(\hat{0}<\gamma_{1}<\cdots<\gamma_{r}<\alpha\right) \otimes\left(\hat{0}<H_{1}<\cdots<H_{s}<G\right)\right) \\
=\hat{0}<\gamma_{1}<\cdots<\gamma_{r}<\alpha<\omega_{1}<\cdots<\omega_{s}<\hat{1},
\end{gathered}
$$

where $\omega_{i}$ is the unique $(G, K)$-digraph which is greater than $\alpha$ and which has the loop at 1 labelled $H_{i}$.

Observe that

$$
\sigma\left(H_{*}([0, \alpha]) \otimes H_{*}(K)\right)=H_{*}([0, \sigma \alpha]) \otimes H_{*}(K) .
$$

So the trace of $\sigma$ acting on $M^{\prime}$ agrees with the trace of $\sigma$ acting on

$$
M^{\prime \prime}=\bigoplus_{\sigma \alpha=\alpha} H_{*}([0, \alpha]) \otimes H_{*}(K) .
$$

Each of the subspaces $H_{*}([0, \alpha]) \otimes H_{*}(K)$ is $\sigma$-invariant so the trace of $\sigma$ acting on $M^{\prime \prime}$ is the sum of the $\sigma$ acting on each individual $H_{*}([0, \alpha]) \otimes H_{*}(K)$.

Fix $\alpha \in \mathscr{I}_{n}^{(1)}(G)$ with $\sigma \alpha=\alpha$, and let $\operatorname{tr}(\alpha)$ denote the trace of $\sigma$ acting on $H_{*}([0, \alpha]) \otimes H_{*}(K)$. It is easy to see that

$$
\operatorname{tr}(\alpha)=\operatorname{tr}\left(\left.\sigma\right|_{H_{*}([0, \alpha])}\right) \otimes \operatorname{tr}\left(\left.\sigma\right|_{H_{*}(K)}\right),
$$

where the action of $\sigma$ on $H_{*}(K)$ depends in some complicated way on $\sigma$ and $\alpha$. It follows from (5.3.3) that $\operatorname{tr}(\alpha)=0$ unless $\sigma$ is $\alpha$-uniform. So we will assume $\sigma$ is $\alpha$-uniform. Thus every cycle of $\sigma$ has the same length $d$ and the same conjugacy type $\mathscr{D}$. By Lemma 5.2.3 we know that

$$
\operatorname{tr}\left(\left.\sigma\right|_{H_{r}([0, \alpha])}\right)=(-1)^{n-1}(-d)^{j-1}(j-1) ! \mu(d) \delta_{r, n-2} .
$$

It remains to compute the trace of $\sigma$ on $H_{*}(K)$.

To understand how $\sigma$ acts on $H_{*}(K)$ it is helpful to redraw Figure 5.2.4 in this situation. Since we have $s=1$ and $t_{1}=1$, we get

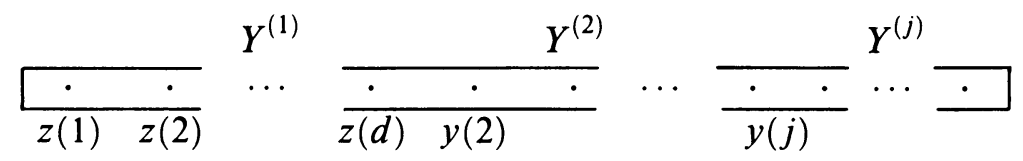


Recall that $\sigma$ is standard. So we may assume that $Y^{(1)}$ has the form

$$
Y^{(1)}=\left(\begin{array}{ccccc}
z(1) & z(2) & z(3) & \cdots & z(d) \\
0 & e & 0 & & 0 \\
\vdots & 0 & e & \cdots & 0 \\
0 & 0 & 0 & & e \\
g & 0 & 0 & & 0
\end{array}\right) .
$$

Let $h$ be the label on the edge from $z(1)$ to $z(2)$ in $\alpha$. Then the edge from $z(i)$ to $z(i+1)$ also has label $h$ for $i=1,2, \ldots, d-1$ by invariance of $\alpha$ under $\sigma$. So the edge from $z(1)$ to $z(d)$ has label $h^{d-1}$.

Let $k \in K$. Under the isomorphism from $C_{*}([0, \alpha]) \otimes C_{*}(K)$ into $M^{\prime}, k$ maps to the unique connected $K$-digraph $\delta$ which satisfies

(1) $\alpha \leq \delta$ in $\Omega_{n}(G, K)$.

(2) The loop of $\delta$ at $z_{1}=1$ is labelled $k$.

By Proposition 2.1.3(d) the loop at $z(d)$ is labelled $h^{-(d-1)} k h^{d-1}$. If we now apply $\alpha$ to $\delta$ we get a new $K$-digraph where the loop at $z(1)$ is labelled

$$
g^{-1} h^{-(d-1)} k h^{d-1} g=h k h^{-1}
$$

the equality $g^{-1} h^{-(d-1)}=h$ coming from Lemma 5.2.5. to

So if $\hat{0}<k_{1}<\cdots<k_{r}<\hat{1}$ is an $r$-chain in $C_{r}(K)$ then $\sigma$ maps this $r$-chain

$$
\hat{0}<\left(h k_{1} h^{-1}\right)<\left(h k_{2} h^{-1}\right)<\cdots<\left(h k_{r} h^{-1}\right)<\hat{1} .
$$

It follows immediately that $\sigma$ acts on $H_{*}(K)$ according to conjugation by $h$.

So if $\sigma$ has $d j$-cycles all of type $g$ then the trace of $\sigma$ on the $(n-2+r)$ th graded piece of $M$ is

$$
(-1)^{n-1} \mu(d)(-d)^{j-1}(j-1) ! \sum_{h^{d}=g} \operatorname{tr}\left(\left.h\right|_{H_{r}(K)}\right),
$$

where the sum is over all $d$ th roots of $g$ in $G$.

It remains to show that this is the value of the induced character given by the right-hand side of Theorem 5.3.2. To prove this we will use the following formula for an induced character which can be found in Feit [6]. Let $\mathscr{M}$ be a subgroup of $\mathscr{N}$ and let $\mathscr{W}$ be an $\mathscr{M}$-module with character $\chi$. Then the value of the induced character $\operatorname{ind}_{\mathscr{M}}^{\mathcal{N}}(\chi)$ on a group element $n \in \mathscr{N}$ is

$$
\operatorname{ind}_{\mathscr{M}}^{\mathscr{N}}(\chi)(n)=\frac{1}{|\mathscr{M}|} \sum_{g \in \mathscr{N}} \chi^{*}\left(g^{-1} n g\right),
$$

where $\chi^{*}(u)$ is $\chi(u)$ if $u \in \mathscr{M}$ and is 0 if $u \notin \mathscr{M}$.

We will apply (5.3.5) in our situation (namely) $\mathscr{M}=C_{n} \times G, \mathscr{N}=\mathscr{G}$, and $\mathscr{W}=\mathbb{C} \otimes H_{*+(n-1)}(K)$. Let $\rho_{r}$ denote the character of $G$ on $H_{r+(n-1)}(K)$. Let $\left(\tau_{n}^{l}, h\right) \in C_{n} \times G$, let $j$ be the greatest common divisor of $l$ and $n$, and let 
$d=n / j$. Then $\left(\tau_{n}^{l}, h\right)$ (as an element of $G \mathrm{wr} S_{n}$ ) consists of $j$ cycles each of length $d$ and type $g=h^{d}$. So by (5.3.5)

$$
\operatorname{ind}_{C_{n} \times G}^{G \text { wr } S_{n}}\left(z_{n} \otimes \rho_{*}\right)(\sigma)=0
$$

unless $\sigma$ consists of $j d$-cycles all of the same type $g$ for some $d \mid n$. If $\sigma$ does consist of $j d$-cycles of type $g$ then

$$
\operatorname{ind}_{C_{n} \times G}^{G \text { wr } S_{n}}\left(z_{n} \otimes \rho_{*}\right)(\sigma)=\frac{\left|z_{\sigma}\right|}{n|G|} \sum_{h^{d}=g} \sum_{(l, n)=j} z_{n}^{l} \rho_{*}(h),
$$

where $z_{\sigma}$ denotes the centralizer of $\sigma$ in $\mathscr{G}$, where the first sum is over all $d$ th roots of $g$ in $G$, and where the second sum is over all $l \in\{1,2, \ldots, n\}$ such that the greatest common divisor of $l$ and $n$ is $d$. Recall that

$$
\sum_{(l, n)=j} z_{n}^{l}=\mu(d) .
$$

Also observe that

$$
\left|z_{\sigma}\right|=(d|G|)^{j} j !=n|G|\left\{(d|G|)^{j-1}(j-1) !\right\} .
$$

So if $\sigma$ consists of $j d$-cycles all of type $g$ then

$$
\begin{aligned}
& \operatorname{sgn}(\sigma) \operatorname{ind}_{C_{n} \times G}^{G \operatorname{wr} S_{n}}\left(\zeta_{n} \otimes \rho_{*}\right)(\sigma) \\
& \quad=(-1)^{(d-1) j} \mu(d)(d|G|)^{j-1}(j-1) ! \sum_{h^{d}=g} \rho_{*}(h) .
\end{aligned}
$$

Since $(-1)^{(d-1) j}=(-1)^{h-1}(-1)^{j-1}$ we have that the right-hand sides of $(5.3 .4)$ and (5.3.6) agree, which completes the proof of our theorem.

5.4. The action of $\mathscr{G}$ on $H_{*}\left(D_{n}(G, K)\right)$. In this section we compute the character of $\mathscr{G}$ and express this character as a sum of induced characters. These characters are induced from centralizers of permutations and are a generalized version of certain characters introduced recently by Reutenauer [14], in one context, and Gerstenhaber and Schack [7] and Loday [12] in quite a different context.

Let $\sigma$ be a permutation in $\mathscr{G}$ having $j_{u} u$-cycles for each $u$. The centralizer of $\sigma$, denoted $z_{\sigma}$, is isomorphic to a direct product over $u$ of $\left(C_{u} \times G\right) \operatorname{wr} S_{j_{u}}$. We will now describe a graded character of $z_{\sigma}$. This character $\pi_{*}^{(\sigma)}$ is a product over $u$ of graded characters $\pi_{*}^{(\sigma)}(u)$ of $\left(C_{u} \times G\right) \operatorname{wr} S_{j_{u}}$. The character $\pi_{*}^{(\sigma)}(u)$ comes from the graded character $z_{u} \otimes \rho_{*}$ on $C_{u} \times G$, where $z_{u}$ is the linear character of $C_{u}$ defined by $z_{u}((1,2, \ldots, u))=\mathscr{C}^{2 \pi i / u}$, and $\rho_{*}$ is the graded character of $G$ on $H_{*}(K)$. The character $z_{u} \otimes \rho_{*}$ is extended to $\left(C_{u} \times G\right) \operatorname{wr} S_{j_{u}}$ using the trivial character on $S_{j_{u}}$. The precise definition of $\pi_{*}^{(\sigma)}(u)$ is

$$
\left(\tau ; \omega_{1}, \omega_{2}, \ldots, \omega_{u}\right) \in\left(C_{u} \times G\right) \operatorname{wr} S_{j_{u}},
$$


where $\tau \in S_{j_{u}}$ and $\omega_{i} \in C_{u} \times G$; then

$$
\pi_{*-(u-1) j_{u}}^{(\sigma)}(u)\left(\tau ; \omega_{1}, \ldots, \omega_{u}\right)=\prod_{\mathscr{C}}\left(z_{u} \otimes \rho_{*}\right)\left(\omega_{C_{1}} \omega_{C_{2}} \cdots \omega_{C_{s}}\right),
$$

where the product on the right is over the cycles $\mathscr{C}=\left(C_{1}, \ldots, C_{s}\right)$ of $\tau$.

We can now state the main result of this section.

Theorem 5.4.1. Let $P$ be a set containing one permutation of each cycle type and let $\beta_{*}^{(n)}(G, K)$ be the graded character of $\mathscr{G}$ on the homology of $D_{n}(G, K)$. Then

$$
\beta_{*}^{(n)}(G, K)=\operatorname{sgn} \cdot \bigoplus_{\sigma \in P} \operatorname{ind}_{Z_{\sigma}}^{G \operatorname{wr} S_{n}}\left(\pi_{*}^{(\sigma)}\right) .
$$

Proof. We begin with formula (4.4.4) from $\S 4.4$ which gives an expression for the $E^{1}$ term in a spectral sequence which abuts to $H_{*}\left(D_{n}(G, K)\right)$. Recall that this spectral sequence collapses at $E^{1}$ and observe that all differentials in this spectral sequence are $\mathscr{G}$-equivariant. So the character $\beta_{*}^{(n)}(G, K)$ is equal to the character of $\mathscr{G}$ acting on

$$
\bigoplus_{\alpha} H_{*}([0, \alpha]) \otimes\left(H_{*}(K)\right)^{\otimes c(\alpha)} .
$$

For each partition $\lambda$ of $n$, let $V_{\lambda}$ denote the subspace of (5.4.2) consisting of the sum of all $H_{*}([0, \alpha]) \otimes\left(H_{*}(K)\right)^{\otimes c(\alpha)}$ over all $\alpha$ which have connected components of sizes $\lambda_{1}, \lambda_{2}, \ldots, \lambda_{l}$. Then $V_{\lambda}$ is a $\mathscr{G}$ module-let $\xi_{\lambda}$ denote the trace of $\mathscr{G}$ acting on $V_{\lambda}$. We will show that

$$
\xi_{\lambda}=\operatorname{sgn} \cdot \operatorname{ind}_{Z_{\sigma}}^{G \operatorname{wr} S_{n}}\left(\Pi_{*}^{(\sigma)}\right),
$$

where the $\sigma$ on the right is the permutation in $P$ with cycle type $\lambda$.

The proof of (5.4.3) is similar to the proof of Theorem 5.3.2. We leave the details to the reader.

It is interesting to note that the induced characters

$$
\operatorname{sgn} \cdot \operatorname{ind}_{z_{\sigma}}^{G \operatorname{wr} S_{n}}\left(\Pi_{*}^{\sigma)}\right), \quad \sigma \in P,
$$

which constitute the summands on the right-hand side of Theorem 5.4.1 have appeared before in the case that $G$ is the 1-element group. These characters of $S_{n}$ were introduced by Reutenauer [14] in connection with the free Lie algebra and were studied further in this context by Garsia, Bergeron, and Bergeron [1]. Independently these same induced characters came up in the study of a Hodge decomposition of Hochschild and cyclic homologies of a commutative algebra $A$ (see Burghelea and Vigué-Poirrier [3], Gerstenhaber and Schack [7], Hanlon [10], and Loday [12]). It is unclear why these induced characters turn up in such varied contexts. 


\section{REFERENCES}

1. F. Bergeron, N. Bergeron, and A. M. Garsia, Idempotents for the free Lie algebra and qenumeration, preprint.

2. A. Bjorner and J. Walker, A homotopy complementation formula for partially ordered sets, European J. Combin. 4 (1983), 11-19.

3. D. Burghelea and M. Vigué-Poirrier, Cyclic homology of commutative algebras, Proc. Meeting on Algebraic Homotopy (Louvain 1986), Lecture Notes in Math., vol. 1318, Springer, 1988.

4. H. H. Crapo, The Möbius function of a lattice, J. Combin. Theory 1 (1966), 126-131.

5. T. A. Dowling, A class of geometric lattices based on finite groups, J. Combin. Theory Ser. B 14 (1973), 61-86.

6. W. Feit, Characters of finite groups, Benjamin, 1967.

7. M. Gerstenhaber and S. Schack, A Hodge-type decomposition for commutative algebra cohomology, J. Pure Appl. Algebras 48 (1987), 229-247.

8. M. Goresky and R. Macpherson, Stratified Morse theory, Springer-Verlag, 1988.

9. P. Hanlon, The fixed-point partition lattices, Pacific J. Math. 96 (1981), 319-341.

10. - The action of $S_{n}$ on the components of the Hodge decomposition of Hochschild homology, Michigan Math. J. 37 (1990), 105-124.

11. G. James and A. Kerber, The representation theory of the symmetric group, Encyclopedia of Math., Vol. 16, Addison-Wesley, New York, 1981.

12. J. L. Loday, Opérations sur l'homologie cyclique des algèbres commutatives, Invent. Math. 96 (1989), 205-230.

13. D. Passman, Permutation groups, Yale Univ. Press, 1967.

14. C. Reutenauer, Theorem on Poincaré-Birkhoff-Witt, logarithm, and representations of the symmetric group whose orders are the Stirling numbers, preprint.

15. G. C. Rota, On the foundations of combinatorial theory I: theory of Mobius functions, Z. Wahrsch. Verw. Gebiete 2 (1964), 340-368.

16. R. P. Stanley, Some aspects of groups acting on finite posets, J. Combin. Theory Ser. A 32 (1982), 132-161.

Department of Mathematics, University of Michigan, Ann Arbor, Michigan 481091003 